\title{
ON THE KUMMER CONGRUENCES AND THE STABLE HOMOTOPY OF $B U$
}

\author{
ANDREW BAKER, FRANCIS CLARKE, NIGEL RAY AND LIONEL SCHWARTZ
}

\begin{abstract}
We study the torsion-free part of the stable homotopy groups of the space $B U$, by considering upper and lower bounds. The upper bound is furnished by the ring $P K_{*}(B U)$ of coaction primitives into which $\pi_{*}^{S}(B U)$ is mapped by the complex $K$-theoretic Hurewicz homomorphism
\end{abstract}

$$
\pi_{*}^{S}(B U) \rightarrow P K_{*}(B U) \text {. }
$$

We characterize $P K_{*}(B U)$ in terms of symmetric numerical polynomials and describe systematic families of elements by utilizing the classical Kummer congruences among the Bernoulli numbers. For a lower bound we choose the ring of those framed bordism classes which may be represented by singular hypersurfaces in $B U$. From among these we define families of classes constructed from regular neighborhoods of embeddings of iterated Thom complexes in Euclidean space. Employing techniques of duality theory, we deduce that these two families correspond, except possibly in the lowest dimensions, under the Hurewicz homomorphism, which thus provides a link between the algebra and the geometry. In the course of this work we greatly extend certain $e$-invariant calculations of J. F. Adams.

\section{INTRODUCTION}

One of the main goals of stable homotopy theory has long been to compute the groups $\pi_{*}^{S}(X)$ for as many examples as possible of spaces and spectra $X$. For a few well-known cases such as the Eilenberg-Mac Lane spectra, various types of $K$-theory spectra, and bordism spectra like $M U$ and its mutants, such computations have been carried out, either by direct construction from the definition or by Adams spectral sequence methods.

For spaces, however, almost no global results are known. A major factor in this situation has been the extreme difficulty of the simplest case, $X=S^{0}$, which defines the coefficient ring $\pi_{*}^{S}\left(S^{0}\right)$, usually abbreviated to $\pi_{*}^{S}$. Systematic knowledge here would allow Atiyah-Hirzebruch spectral sequence techniques to be brought to bear on the general case.

Mosher's paper [36] is one of the few studies with which we are familiar which carries out specific calculations along these lines; it deals with the case of infinite-dimensional complex projective space, $X=C P^{\infty}$.

Received by the editors May 20, 1988.

1980 Mathematics Subject Classification (1985 Revision). Primary 55R45; Secondary 55N15, 55Q10, 19L64, 11B68.

Key words and phrases. Stable homotopy groups, Bernoulli numbers, $K$-theory. 
Of course, the Adams and Novikov spectral sequences are always lurking in the shadows, awaiting use by the bold. However, in many cases the algebraic complexity which they embody is enormous and, we suggest, may obscure the more geometric aspects of the space at hand.

One of the few satisfactory investigations of $\pi_{*}^{S}$ has been the determination of the groups $\operatorname{Im} J$, mainly by Adams [2]. These constitute a cyclic direct summand of order $m(2 n)$ in each dimension $4 n-1$, where $m(2 n)$ is the denominator of the divided Bernoulli number $B_{2 n} / 4 n$ expressed in its lowest terms.

At one time it seemed plausible that the geometrical description, pioneered by L. S. Pontrjagin, of $\pi_{k}^{S}(Y)$ as the framed bordism group $\Omega_{*}^{\mathrm{fr}}(X)$, might herald a significant breakthrough. But experience has shown that, in the case of the coefficient ring, it is exceedingly difficult to assemble examples of nonbounding framed manifolds.

There is, however, a chink in the armor for the case of a general space $X$. We may map framed boundaries into $X$ and still hope to obtain nonzero classes in the reduced groups $\widetilde{\Omega}_{*}^{\mathrm{fr}}(X)$. The simplest example of this idea is suggested by $\operatorname{Im} J$, which measures bordism classes of framed spheres. Thus, if we map spheres into $X$, we are examining the image of the unstable groups $\pi_{*}(X)$ in $\pi_{*}^{S}(X)$.

Our strategy here is to investigate singular manifolds which, while still framed boundaries, are a stage more complicated. They are, in fact, hypersurfaces, that is, codimension one submanifolds of spheres. Each may be constructed by embedding a finite $C W$-complex $Y$ in a sphere. By this procedure we are actually examining the image of $\pi_{*}\left(\Omega\left(S^{1} \wedge X\right)\right)$ in $\pi_{*}^{S}(X)$ under the stabilization map.

Our study concerns the case of the classifying space of the infinite unitary group, $X=B U$. We shall show, in the geometric part of our work, that the above method yields much information. In the main this involves the complex $Y$ being an iterated Thom complex, a type of space with many useful properties.

One of our hopes is that, by exhibiting this approach in action, others may find it applicable to their own choice of spaces.

Of course, having evolved such a process, we require a detection procedure for the classes so created. Here enters complex $K$-theory.

For any $X$, the Hurewicz homomorphism

$$
\pi_{*}^{S}(X) \rightarrow K_{*}(X)
$$

has image lying in the subgroup $P K_{*}(X)$ of elements which are primitive with respect to the coaction of the cooperation algebra $K_{*}(K)$. Indeed, if $X$ is the spectrum $M U$ the Hattori-Stong theorem tells us that this map to the primitives is an isomorphism. This is also the case, modulo torsion, for the space $X=C P^{\infty}$. Thus the primary topic of the algebraic part of our work is the investigation of $P K_{*}(B U)$. Since $B U$ is a loop space, all the groups involved are in fact rings, via the Pontrjagin product. 
It is in the context of $P K_{*}(B U)$ that we encounter the Kummer congruences, although we require a considerable amount of preliminary algebra to make the connection. From the time of their discovery in 1851 [32], these congruences remained something of a mystery until Kubota and Leopoldt, and later Mazur, showed how they follow in a simple fashion from the existence of a certain $p$-adic measure [30]. We show here that the Kummer congruences, in the form in which they were originally introduced, rather than their later generalizations, fit naturally into algebraic topology.

For us the congruences are encapsulated by the existence of a transformation from $K_{*}\left(C P^{\infty}\right)$ to $K_{*}(K)$. This transformation is closely related to the Thom isomorphism in $K$-theory and may be used to obtain families of elements in $P K_{*}(B U)$. Moreover, in this guise we are able to give a new, purely algebraic proof of the congruences which may be of some interest in its own right. A particular feature of this approach is that we are able to describe phenomena globally, i.e., for all primes simultaneously, rather than for one prime at a time.

It should by now be no great surprise to discover that the primitives obtained by iterating the Kummer congruences are exactly the Hurewicz images of the stable classes created out of embeddings of the iterated Thom complexes. Thus do the algebra and geometry finally combine. Since this point is the crux of our thesis, we shall briefly digress to detail the simplest case. Here, as throughout, we write $\Sigma_{i}$ for the Bott generator of $\pi_{2 i}(B U)$, as well as for its stabilization in $\pi_{2 i}^{S}(B U)$ and its Hurewicz image in $K_{2 i}(B U)$.

A two-cell iterated Thom complex has the form $S^{2 i} \cup_{\alpha} e^{2(i+j)}$, where $\alpha$ generates the image of the complex $J$-homomorphism, and so has order $\frac{1}{2} m(j)$. Embedding in $S^{2(i+j+k)+1}$ for suitably large $k$, and mapping the resulting hypersurface into $B U$, yields an element of $\pi_{2(i+j+k)}^{S}(B U)$ whose Hurewicz image we compute to be

$$
\left(B_{j} / j\right)\left(\Sigma_{i+j} \Sigma_{k}-\Sigma_{i} \Sigma_{j+k}\right) .
$$

This is an element of $P K_{*}(B U)$ which is given by a single application of the Kummer congruences. Its existence is effectively equivalent to the von Staudt theorem on the denominator of $B_{j} / j$.

We deduce that in $\pi_{2(i+j+k)}^{S}(B U)$, the class $\Sigma_{i+j} \Sigma_{k}-\Sigma_{i} \Sigma_{j+k}$ is divisible by $m(j) / 2$ modulo torsion. In fact, in this and in certain other cases we can actually establish that this divisibility holds precisely.

As we iterate these procedures we obtain a family of even more intricate divisibility statements in $\pi_{*}^{S}(B U)$; their complexity is, however, exactly tabulated by the iterated congruences. It transpires that the numerators of the divided Bernoulli numbers $B_{j} / j$, as well as their denominators, play a leading role. This suggests that, in the past, too much attention has been paid to the function $m(j)$ rather than to the numbers $B_{j} / j$ themselves.

Throughout our work the geometry and algebra are obviously closely intertwined, and we have so far found it easier to describe the geometry first by way 
of motivation. In the main body of the text, on the other hand, it has seemed more natural to get to grips with $P K_{*}(B U)$ first and regard the iterated Thom complex construction as a realization process for the primitives already introduced. In any case the families of primitives which we construct exist in slightly lower dimensions than those in which we are able to realize them. A further point of view would be to present both the algebraic and geometric aspects simultaneously, but, since we feel that each may have independent interest, we have avoided this course.

Our computations could easily be adapted so as to apply to more general complex-oriented homology theories $E_{*}()$, and so to study the Hurewicz homomorphism

$$
\pi_{*}^{S}(B U) \rightarrow E_{*}(B U) .
$$

The Bernoulli and Stirling numbers would be replaced by their generalized versions; see [35], [9], and [42]. Readers may wish to try this for themselves as and when they feel it useful.

There is, however, one reason why $K_{*}(B U)$ is a particularly important blending of the stable and unstable. There is a case to be made for considering it as an unstable cooperation ring for $K$-theory. This perspective is related to Boardman's [12] and puts several of our results in an attractive context.

Developing this theme, and writing $\Omega^{\infty} E_{\infty}$ for the zero space in the $\Omega$ spectrum of $E$, we could study the Hurewicz homomorphsim

$$
\pi_{*}^{S}\left(\Omega^{\infty} E_{\infty}\right) \rightarrow E_{*}\left(\Omega^{\infty} E_{\infty}\right)
$$

by our methods. That this would produce results in the universal case $E=M U$ follows from the work of Ravenel and Wilson [41].

The most general setting of all would be to examine $F_{*}\left(\Omega^{\infty} E_{\infty}\right)$ for some other complex-oriented theory $F_{*}()$. But this is uncharted water, which we have managed to steer away from!

We now outline the contents of each section.

In $\S 1$ we characterize the $K$-homology of the classifying space of a torus by identifying $K_{0}(B T)$ with the ring of rational polynomials which are integervalued on the integers. We refer to such polynomials as numerical. We further identify the primitive elements, under the coaction of $K_{0}(K)$ on $K_{0}(B T)$, with the subring consisting of those numerical polynomials which are homogeneous. Most of this algebra has recently become folklore. It is, however, surprisingly intricate, as we demonstrate by constructing a $p$-local basis for the ring of homogeneous numerical polynomials in two variables, thus completing a project begun by Knapp [29].

In $\S 2$ we turn our attention to $K_{0}(B U(n))$, which we are now well prepared to describe as the group of symmetric polynomials satisfying a certain integrality condition. Once more, the primitive elements correspond to the homogeneous polynomials. Since the Bott generators of the unstable homotopy groups of 
$B U$ play, as might be expected, a major role in our stable calculations, we give formulas for their $K$-theory Hurewicz images.

$\S 3$ is purely technical and is devoted entirely to algebraic procedures which are required in $\S \S 4$ and 5 . We introduce a certain sequence of recursively defined polynomials and describe their elementary properties.

We are now in a position to commence our program of defining the family of primitives $\Xi_{i, j, K}$ in $K_{0}(B U)$. Thus number theory predominates in $\S 4$, where we are concerned with the construction of numerical polynomials in a single variable. The key ingredient is Theorem 4.3 , which is a global version of the generalized Kummer congruences, and whose proof is inspired by the geometry underlying the space $B U$. This leads us to certain criteria, formulated in terms of admissible sequences, for ensuring that the polynomials constructed along the lines of $\S 3$ are numerical. Such polynomials generalize, and provide an alternative approach to, some congruences of J. F. Adams. We explain the connection at the end of $\S 4$.

It is then possible, exploiting the relationship established in $\S \S 1$ and 2 between numerical polynomials and $K_{0}(B U(n))$, to develop these considerations into the construction of the elements $\Xi_{i, j, K}$; this we explain in $\S 5$. In so doing, we introduce certain other elements in $K_{0}(B U)$ which are not primitive. These are required in order to realize the $\Xi_{i, j, K}$ as stable homotopy classes. This realization is the goal of the next three sections.

In $\S 6$ we show that the global Kummer congruences are intimately related to the $K$-theory Thom isomorphism; the parallel is in fact apparent in our proof of the congruences. We are thus led to define the notion of an iterated Thom complex, by analogy with the process of iterating the congruences. We show that, for each such complex, the $K$-theory slant products of basis elements are represented by the nonprimitive elements of $K_{0}(B U)$ introduced in $\S 5$. This entails the derivation of a formula for the behavior of slant products under the Thom isomorphism. The results of $\S 6$ are used in $\S 8$.

Our strategy for constructing elements $\pi_{*}^{S}(B U)$ is as follows. Given an embedding of a finite complex $Y$ in a sphere $S^{2 n+1}$, let $M$ be the boundary of a regular neighborhood of $Y$, selected so as to be a smooth manifold. As a hypersurface $M$ is framed, so that each map $M \rightarrow B U$ determines a stable homotopy class in $\pi_{2 n}^{S}(B U)$. In $\S 7$ we show how to compute the $K$-theory Hurewicz image of such a class by using $S$-duality, in the case that $K_{0}(Y)$ is torsion-free.

We implement this strategy in $\S 8$ by choosing $Y$ to be an iterated Thom complex. We show that $\Xi_{i, j, K}$ is stably spherical for each strictly admissible sequence $K$, although $K$ need only be admissible for $\Xi_{i, j, K}$ to exist as a primitive in $K_{0}(B U)$. Our realization results imply that certain quadratic expressions in the Bott generators are divisible modulo torsion in $\pi_{*}^{S}(B U)$. In the latter part of $\S 8$ we give a partial answer to the question of whether this divisibility holds exactly. 
In $\S 9$, to show that our methods may be applied in other cases, we define a second family of primitives and deduce that these too may be realized spherically, at least in sufficiently large dimensions. We utilize the results of $\S 7$ in the case where $Y$ is a complex projective space, and as an aside we note that a homotopy nonembedding result for $C P^{n}$ can be read off from our computations.

Our work might suggest that we believe that the stable $K$-theory Hurewicz map is an epimorphism. However, in $\S 10$ we give a 2-primary counterexample to such a conjecture. Also in $\S 10$, by considering the case where $Y$ is a two-cell complex in the construction of $\S 7$, we describe how to improve our realization results in certain low-dimensional cases.

In the concluding $\S 11$, we offer a few tentative conjectures and discuss a variety of questions which, we believe, would repay further investigation.

This paper has taken a long time to prepare and has often been referred to as "in preparation." We can now only apologize for the delay, which has been due, at least in part, to the geographic separation of the authors.

We should thank Sam Gitler for unwittingly setting the whole project in motion in 1979 and Dieter Puppe for helping us to locate [18] at Oberwolfach in 1985.

\section{THE $K$-THEORY OF THE CLASSIFYING SPACES OF TORI}

The spaces $C P^{\infty}, B T^{n}, B U(n)$, and $B U$ are of course extremely familiar and their $K$-theory has been known from the earliest times. However, we shall give a description of their $K$-homology which makes the coaction of the cooperation algebra $K_{*}(K)$, and hence the primitive submodule, easy to understand. This section is concerned with $C P^{\infty}$ and $B T^{n}$. The classifying spaces of the unitary groups will be dealt with in $\S 2$.

Most of the spaces encountered in this paper have cells in even dimensions only. Because of the periodicity of $K$-theory we may, therefore, consider only the group in degree zero. For such a space $X, K_{*}(X)=\pi_{*}(K) \otimes K_{0}(X)$.

We write $t \in \pi_{2}(K)$ for the Bott generator and $u$ and $v \in K_{2}(K)$ for its image under the left and right unit maps, respectively; see [5]. Then if $\alpha \in K_{0}(X)$ and $t^{m} \alpha \in K_{2 m}(X)$ is primitive, $1 \otimes t^{m} \alpha=\psi\left(t^{m} \alpha\right)=u^{m} \psi(\alpha)$ [3]. But $1 \otimes t^{m} \alpha=v^{m} \otimes \alpha$ [3], so that $\psi(\alpha)=w^{m} \otimes \alpha$, where $w=u^{-1} v \in K_{0}(K)$. We therefore define the group

$$
P_{m} K_{0}(X)=\left\{\alpha \in K_{0}(X): \psi(\alpha)=w^{m} \otimes \alpha\right\},
$$

which is isomorphic to the group of primitive elements in dimension $2 m$. Thus the Hurewicz map factors as

$$
\pi_{2 m}^{S}(X) \rightarrow P_{m} K_{0}(X) .
$$

If $X$ is an $H$-space, so that $K_{*}(X)$ and $K_{0}(X)$ are rings, then the coaction maps are ring homomorphisms. Thus the set of primitive elements of $K_{*}(X)$ forms a subring which is isomorphic to the graded subring $P_{*} K_{0}(X)$ of $K_{0}(X)$. 
At the center of this paper is the result 1.3, first proved in [15] and [46], that the $K$-homology of the space $C P^{\infty}$ as Pontrjagin ring (with product induced from the tensor product map) is isomorphic to the ring of numerical polynomials in one variable.

Definition 1.1. A polynomial $f(w) \in \mathbf{Q}[w]$ is said to be numerical if $f(n)$ is an integer for every integer $n$. We let $A$ denote the set of all numerical polynomials.

Clearly $A$ is a subring of $\mathbf{Q}[w]$ which contains $\mathbf{Z}[w]$.

Proposition 1.2. Let

$$
\left(\begin{array}{l}
w \\
n
\end{array}\right)=\frac{w(w-1) \cdots(w-n+1)}{n !}
$$

denote the binomial coefficient polynomial; then $\left(\begin{array}{c}w \\ n\end{array}\right) \in A$ and $\left\{1=\left(\begin{array}{c}w \\ 0\end{array}\right),\left(\begin{array}{l}w \\ 1\end{array}\right)\right.$, $\left.\left(\begin{array}{c}w \\ 2\end{array}\right), \ldots\right\}$ is a basis for $A$ as $\mathbf{Z}$-module.

Proof. This is an elementary exercise in the use of the difference operator; see Lemma 5.1 of [5].

Proposition 1.3. The ring $A$ is isomorphic to $K_{0}\left(C P^{\infty}\right)$, where $K_{0}\left(C P^{\infty}\right)$ has the ring structure induced by the map $C P^{\infty} \times C P^{\infty} \rightarrow C P^{\infty}$ which classifies the tensor product of line bundles.

Proof. Let $x \in H^{2}\left(C P^{\infty}\right)$ be a generator; then the Chern character

$$
\text { ch: } K^{0}\left(C P^{\infty}\right) \rightarrow H^{* *}\left(C P^{\infty} ; \mathbf{Q}\right)=\mathbf{Q}[[x]]
$$

identifies $K^{0}\left(C P^{\infty}\right)$ as $\mathrm{Z}\left[\left[e^{x}-1\right]\right], e^{x}$ being the Chern character of the Hopf bundle [8].

Let $w \in H_{2}\left(C P^{\infty}\right)$ be dual to $x$; then, under the Kronecker product, $\left\langle x^{n}, w^{n}\right\rangle=n !$. The homology Chern character

$$
\operatorname{ch}: K_{0}\left(C P^{\infty}\right) \rightarrow H_{*}\left(C P^{\infty} ; \mathbf{Q}\right)=\mathbf{Q}[w]
$$

will identify $K_{0}\left(C P^{\infty}\right)$ as the subring of $\mathbf{Q}[w]$ which consists of those polynomials $f(w)$ which pair integrally with $\left(e^{x}-1\right)^{n}$ for all $n \geq 0$. This is equivalent to requiring that $\left\langle e^{n x}, f(w)\right\rangle \in \mathbf{Z}$ for all integers $n$. But now, if $f(w)=\sum_{i} a_{i} w^{i}$,

$$
\left\langle e^{n x}, f(w)\right\rangle=\sum_{i, j}\left\langle\frac{n^{j} x^{j}}{j !}, a_{i} w^{i}\right\rangle=\sum_{i} a_{i} n^{i}=f(n) .
$$

Thus $f(w) \in K_{0}\left(C P^{\infty}\right)$ if and only if $f(n)$ is an integer for each integer $n$ as required.

Now

$$
\left\langle\left(e^{x}-1\right)^{n},\left(\begin{array}{l}
w \\
k
\end{array}\right)\right\rangle=\sum_{i=0}^{n}(-1)^{n-i}\left(\begin{array}{l}
n \\
i
\end{array}\right)\left(\begin{array}{l}
i \\
k
\end{array}\right)
$$


If $k>n$ every term in this expansion is zero, while if $k<n$ the expression also equals zero since, expanded as a power series in $x,\left(e^{x}-1\right)^{n}$ contains terms of degree $n$ and above, while $\left(\begin{array}{c}w \\ k\end{array}\right)$ has degree $k$. Clearly $\left\langle\left(e^{x}-1\right)^{n},\left(\begin{array}{c}w \\ n\end{array}\right)\right\rangle=1$.

Thus the binomial coefficient polynomials $\left(\begin{array}{c}w \\ n\end{array}\right)$ are dual to the powers of $e^{x}-1$. Therefore, in the notation of [4], $\left(\begin{array}{c}w \\ n\end{array}\right)=\beta_{n}$. We shall use both notations for this element.

We let $\beta_{k}$ denote also the image of $\beta_{k}=\left(\begin{array}{c}w \\ k\end{array}\right)$ under the inclusion

$$
i_{*}: K_{0}\left(C P^{\infty}\right)=K_{0}(B U(1)) \rightarrow K_{0}(B U) .
$$

The elements $\beta_{1}, \beta_{2}, \ldots$ are polynomial generators, with respect to the product induced by the Whitney sum, for the ring $K_{0}(B U)$ [4].

$K_{0}(K)$ may be defined as the direct limit of the system

$$
K_{0}(B U) \stackrel{B_{*}}{\longrightarrow} K_{0}(B U) \stackrel{B_{*}}{\longrightarrow} K_{0}(B U) \stackrel{B_{*}}{\longrightarrow} \cdots
$$

under the Bott map $B_{*}: K_{0}(B U) \rightarrow K_{0}(B U)$. By Lemma 6.11 of [5] or Corollary 17.27 of [52], $B_{*}\left(\beta_{k}\right) \equiv k \beta_{k}+(k+1) \beta_{k+1}$ modulo decomposables, while $B_{*}$ annihilates decomposables.

Consider the diagram

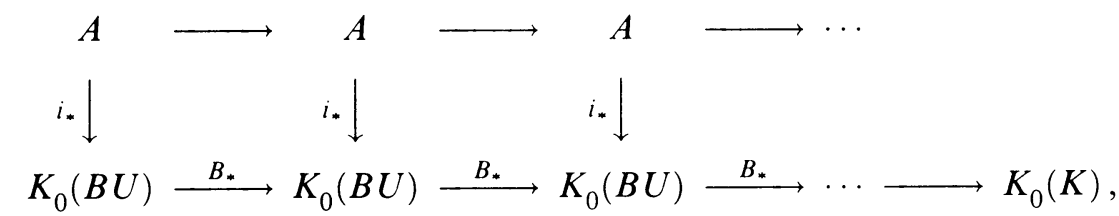

where the maps $A \rightarrow A$ are multiplication by $w$. It follows from the identity

$$
w\left(\begin{array}{l}
w \\
k
\end{array}\right)=k\left(\begin{array}{l}
w \\
k
\end{array}\right)+(k+1)\left(\begin{array}{c}
w \\
k+1
\end{array}\right)
$$

that although each square does not commute it does so after the application of another Bott map. This is sufficient to induce a map in the limit

$$
A\left[w^{-1}\right] \rightarrow K_{0}(K),
$$

which it is now easy to check is an isomorphism; in fact it is an isomorphism of rings, both ring structures being induced from the tensor product of vector bundles.

This gives a simplified proof of the unitary case of the main result of [5].

We refer to elements of $A\left[w^{-1}\right]$ as stably numerical polynomials. They are characterized as those rational Laurent polynomials $f(w)$ such that $f(n) \in$ $\mathbf{Z}[1 / n]$ for every nonzero integer $n$. For every stably numerical polynomial $f(w)$ there exists $k \geq 0$ such that $w^{k} f(w)$ is numerical.

Since the natural map $C P^{\infty} \rightarrow K$ induces the inclusion $A \subset A\left[w^{-1}\right]$ and the diagonal of the Hopf algebra $K_{0}(K)=A\left[w^{-1}\right]$ maps $w$ to $w \otimes w$, the coaction

$$
\psi: K_{0}\left(C P^{\infty}\right) \rightarrow K_{0}(K) \otimes K_{0}\left(C P^{\infty}\right)
$$

is also given by the ring homomorphism $w \mapsto w \otimes w$. 
Thus, for example, the image of $\beta_{3}=\left(\begin{array}{l}w \\ 3\end{array}\right)$ under the coaction is $\left(\begin{array}{c}w \otimes w \\ 3\end{array}\right)$, which, using the identity

$$
\begin{aligned}
\left(\begin{array}{c}
x y \\
3
\end{array}\right)= & \left(\begin{array}{l}
x \\
3
\end{array}\right)\left(\begin{array}{l}
y \\
1
\end{array}\right)+4\left(\begin{array}{l}
x \\
2
\end{array}\right)\left(\begin{array}{l}
y \\
2
\end{array}\right)+\left(\begin{array}{l}
x \\
1
\end{array}\right)\left(\begin{array}{l}
y \\
3
\end{array}\right) \\
& +6\left(\begin{array}{l}
x \\
3
\end{array}\right)\left(\begin{array}{l}
y \\
2
\end{array}\right)+6\left(\begin{array}{l}
x \\
2
\end{array}\right)\left(\begin{array}{l}
y \\
3
\end{array}\right)+6\left(\begin{array}{l}
x \\
3
\end{array}\right)\left(\begin{array}{l}
y \\
3
\end{array}\right),
\end{aligned}
$$

gives (we think of $x$ as $w \otimes 1$ and $y$ as $1 \otimes w$ )

$$
\begin{aligned}
\psi\left(\beta_{3}\right)= & \left(\begin{array}{c}
w \\
3
\end{array}\right) \otimes \beta_{1}+\left(4\left(\begin{array}{c}
w \\
2
\end{array}\right)+6\left(\begin{array}{c}
w \\
3
\end{array}\right)\right) \otimes \beta_{2} \\
& +\left(\left(\begin{array}{c}
w \\
1
\end{array}\right)+6\left(\begin{array}{c}
w \\
2
\end{array}\right)+6\left(\begin{array}{c}
w \\
3
\end{array}\right)\right) \otimes \beta_{3} .
\end{aligned}
$$

Similarly, a numerical polynomial $f(w) \in A$ will represent an element of $P_{m} K_{0}\left(C P^{\infty}\right)$ if and only if it satisfies

$$
f(w \otimes w)=w^{m} \otimes f(w),
$$

which implies that $f(w)$ is an integer multiple of $w^{m}$.

Since $w \in P_{1} K_{0}\left(C P^{\infty}\right)$ is realized by the homotopy class $S^{2}=C P^{1} \subset C P^{\infty}$ which we denote by $\Gamma_{1}$, it follows that the Hurewicz map

$$
\pi_{*}^{S}\left(C P^{\infty}\right) \rightarrow P_{*} K_{0}\left(C P^{\infty}\right)
$$

is onto, with $w^{m} \in P_{m} K_{0}\left(C P^{\infty}\right)$ realized by $\left(\Gamma_{1}\right)^{m} \in \pi_{2 m}^{S}\left(C P^{\infty}\right)$.

This confirms Mosher's result, Theorem 2.1 of [36], that $\pi_{*}^{S}\left(C P^{\infty}\right)$ modulo torsion is a polynomial ring on $\Gamma_{1}$. We will see below that the situation is more complicated for $\pi_{*}^{S}(B U)$.

Definition 1.5. A polynomial $f\left(w_{1}, \ldots, w_{n}\right) \in \mathbf{Q}\left[w_{1}, \ldots, w_{n}\right]$ is numerical if $f\left(k_{1}, \ldots, k_{n}\right) \in \mathbf{Z}$ for every $\left(k_{1}, \ldots, k_{n}\right) \in \mathbf{Z}^{n}$. The numerical polynomials in $n$ variables form a ring, which we denote by $A_{n}$.

Proposition 1.6. The ring $A_{n}$ is isomorphic to the iterated tensor product $A^{\otimes n}$ and hence has a basis consisting of the elements

$$
\left(\begin{array}{l}
w_{1} \\
k_{1}
\end{array}\right)\left(\begin{array}{l}
w_{2} \\
k_{2}
\end{array}\right) \ldots\left(\begin{array}{l}
w_{n} \\
k_{n}
\end{array}\right), \quad k_{1}, k_{2}, \ldots, k_{n} \geq 0 .
$$

Proof. See $\S 26.1$ of [25], or [14, $\S 12$, no. 4, Proposition 12]. The essential idea is that the proof of Proposition 1.2 generalizes to show that $A_{n} \cong A_{n-1} \otimes A$.

Corollary 1.7. If $B T^{n}=\left(C P^{\infty}\right)^{n}$ denotes the classifying space of an $n$-torus then $K_{0}\left(B T^{n}\right)$ is isomorphic to the ring $A_{n}$ of numerical polynomials in $n$ variables. The coaction

$$
\psi: K_{0}\left(B T^{n}\right) \rightarrow K_{0}(K) \otimes K_{0}\left(B T^{n}\right)
$$

is the ring homomorphism determined by $\psi\left(w_{i}\right)=w \otimes w_{i}$.

Proof. Use the Künneth formula and naturality of the coaction map. 
Proposition 1.8. The group of primitive elements $P_{m} K_{0}\left(B T^{n}\right)$ is isomorphic to the group of numerical polynomials in $n$ variables which are homogeneous of degree $m$.

Proof. The condition that $f\left(w_{1}, \ldots, w_{n}\right) \in P_{m} K_{0}\left(B T^{n}\right)$ is that

$$
f\left(w \otimes w_{1}, \ldots, w \otimes w_{n}\right)=w^{m} \otimes f\left(w_{1}, \ldots, w_{n}\right) .
$$

In other words, $f$ is homogeneous of degree $m$.

The next proposition gives us a method of constructing homogeneous numerical polynomials.

Proposition 1.9. Suppose that $f\left(w_{1}, \ldots, w_{n-1}\right) \in A_{n-1}$ has total degree $k$ and denominator $M$, so that $M f\left(w_{1}, \ldots, w_{n-1}\right)$ has integer coefficients; then, for $j$ sufficiently large, $w_{n}^{k+j} f\left(w_{1} w_{n}^{-1}, \ldots, w_{n-1} w_{n}^{-1}\right)$ is a homogeneous numerical polynomial of degree $k+j$.

In fact $w_{n}^{k+j} f\left(w_{1} w_{n}^{-1}, \ldots, w_{n-1} w_{n}^{-1}\right)$ is numerical if $j$ is greater than or equal to the maximum exponent of any prime occurring in $M$.

Proof. Let $g\left(w_{1}, \ldots, w_{n-1}\right)$ denote the numerator $M f\left(w_{1}, \ldots, w_{n-1}\right)$ so that $w_{n}^{k} g\left(w_{1} w_{n}^{-1}, \ldots, w_{n-1} w_{n}^{-1}\right)$ is a homogeneous integer polynomial. We need to show that if $p$ is prime, $p^{e}$ divides $M$ and $j \geq e$, then

$$
a_{n}^{k+j} g\left(a_{1} / a_{n}, \ldots, a_{n-1} / a_{n}\right) \equiv 0 \bmod p^{e},
$$

for all integers $a_{1}, \ldots, a_{n}$.

If $p$ divides $a_{n}$ then $p^{e}$ divides $a_{n}^{j}$ and the congruence holds.

Otherwise we choose $b$ so that $a_{n} b \equiv 1 \bmod p^{e}$, then

$$
a_{n}^{k+j} g\left(a_{1} / a_{n}, \ldots, a_{n-1} / a_{n}\right) \equiv a_{n}^{k+j} g\left(a_{1} b, \ldots, a_{n-1} b\right) \bmod p^{e} .
$$

But $p^{e}$ divides any value of $g$ since $f$ is numerical.

The estimate for $j$ in this proposition is not, in general, the best possible. Considering the case $n=2$, where we write $x=w_{1}$ and $y=w_{2}$, it is clear that any homogeneous numerical polynomial $g(x, y)$ of degree $r$ can be written as $y^{r} f\left(x y^{-1}\right)$, for a unique numerical polynomial in a single variable defined by $f(w)=g(w, 1)$. If $f(w)$ has degree $k$ it may happen that $r-k$ is less than the largest prime exponent in $M$, the denominator of $f(w)$. The polynomials $q_{i}(x, y)$ defined below are examples, for $i>1$. If, however, $M f(w)$ is monic and a prime power $p^{e}$ divides $M$, consideration of the value of $M g(1, p)$ modulo $p^{e}$ shows that $r$ must be at least $k+e$.

To demonstrate the nontrivial nature of the graded ring of homogeneous numerical polynomials we proceed now to give a $p$-local basis, for any prime $p$, in the two-variable case. This problem has also been considered by Knapp in [29].

Fix a prime $p$. Let $h(x, y)$ be a homogeneous integer polynomial of degree $p^{2}-1$ such that for all integers $a$ and $b$ which are not both divisible by $p$ we 
have $h(a, b) \equiv 1 \bmod p$. For example, let $h(x, y)=x^{p^{2}-1}-x^{p^{2}-p} y^{p-1}+y^{p^{2}-1}$, or, if $p$ is an odd prime, $h(x, y)=x^{p^{2}-1}-x^{\left(p^{2}-1\right) / 2} y^{\left(p^{2}-1\right) / 2}+y^{p^{2}-1}$, which has the advantage of symmetry. by

Given $h(x, y)$, we define the sequence of polynomials $q_{k}(x, y)$ recursively

$$
\begin{aligned}
& q_{1}(x, y)=\left(x y^{p}-x^{p} y\right) / p, \\
& q_{k}(x, y)=\left(q_{k-1}(x, y)^{p}-h(x, y)^{p^{k-2}} q_{k-1}(x, y)\right) / p, \quad \text { if } k>1 .
\end{aligned}
$$

It is clear that $q_{k}(x, y)$ is a homogeneous polynomial of degree $p^{k}+p^{k-1}$, and its denominator is $p^{p^{k-1}+p^{k-2}+\cdots+p+1}$. It follows that if $p$ divides both $a$ and $b$ then $q_{k}(a, b)$ is an integer; in fact it is divisible by $p$. Fermat's theorem, together with the property we have assumed for $h(x, y)$, shows inductively that $q_{k}(a, b)$ is an integer for all other integral values of $a$ and $b$. Thus $q_{k}(x, y)$ is a numerical polynomial.

If $p$ is odd and a symmetric choice is made for $h(x, y)$ then $q_{k}(x, y)$ is antisymmetric for all $k \geq 1$.

Given integers $m$ and $n$, with $0 \leq m \leq n$, let $m=(p+1) t+r$, with $0 \leq r<p+1$, and write $t=t_{1}+t_{2} p+\cdots+t_{k} p^{k-1}$, where $0 \leq t_{i}<p$. We define

$$
g_{m}^{n}(x, y)= \begin{cases}x^{n-m} y^{r} q_{1}(x, y)^{t_{1}} q_{2}(x, y)^{t_{2}} \cdots q_{k}(x, y)^{t_{k}}, & \text { if } r<p, \\ y^{n-m+r} q_{1}(x, y)^{t_{1}} q_{2}(x, y)^{t_{2}} \cdots q_{k}(x, y)^{t_{k}}, & \text { if } r=p,\end{cases}
$$

so that $g_{m}^{n}(x, y)$ is a homogeneous numerical polynomial of degree $n$.

Lemma 1.10. If $f(x, y)=\sum_{m=0}^{n} \lambda_{m} g_{m}^{n}(x, y)$ where the coefficients $\lambda_{m}$ are integers and $f(a, b) \equiv 0 \bmod p$ for all integers $a$ and $b$, then $p$ divides each coefficient $\lambda_{m}$.

Proof. We use induction on $k$, the largest index such that $q_{k}(x, y)$ appears in $f(x, y)$ with a nonzero coefficient.

If $k=0$ then $f(x, y)=\sum_{m=0}^{p-1} \lambda_{m} x^{n-m} y^{m}+\lambda_{p} y^{n}$. The coefficient $\lambda_{p}=$ $f(0,1)$ is divisible by $p$, while $f(1, y)-f(0,1) y^{n}$ is a polynomial of degree at most $p-1$ which has $p$ distinct roots modulo $p$, and so it must be the zero polynomial modulo $p$.

For $k>0$ we write $f(x, y)=\sum_{i=0}^{p-1} f_{i}(x, y) q_{k}(x, y)^{i}$, where $f_{i}(x, y)=$ $\sum_{m=0}^{K-1} \lambda_{m+i K} g_{m}^{n-i K}(x, y)$ with $K=p^{k}+p^{k-1}$ and $\lambda_{j}=0$ for $j>n$.

The induction step will be completed if we can show that, for each $i, p$ divides $f_{i}(a, b)$ for all integers $a$ and $b$.

Since $p$ divides $q_{j}(a, b)$ if both $a$ and $b$ are multiples of $p$ it follows that $f_{i}(a, b)$ is certainly divisible by $p$ in this case.

If $d$ is an integer $\left(a+d p^{k}\right)^{p} \equiv a^{p} \bmod p^{k+1}$. It follows that $q_{1}\left(a+d p^{k}, b\right) \equiv$ $q_{1}(a, b)+d b^{p} p^{k-1} \bmod p^{k}$. This implies that $q_{1}\left(a+d p^{k}, b\right) \equiv q_{1}(a, b) \bmod$ $p^{k-1}$, so that $q_{1}\left(a+d p^{k}, b\right)^{p} \equiv q_{1}(a, b)^{p} \bmod p^{k}$, which gives $q_{2}\left(a+d p^{k}, b\right) \equiv$ 
$q_{2}(a, b)-h(a, b) d b^{p} p^{k-2} \bmod p^{k-1}$. We use here the fact that $h\left(a+d p^{k}, b\right) \equiv$ $h(a, b) \bmod p^{k}$ since $h(x, y)$ is an integer polynomial.

Iterating this argument we have

$$
q_{j}\left(a+d p^{k}, b\right) \equiv q_{j}(a, b)+(-1)^{j-1} h(a, b)^{1+p+\cdots+p^{j-2}} d b^{p} p^{k-j} \bmod p^{k-j+1},
$$

for $j \leq k$.

Thus $q_{j}\left(a+d p^{k}, b\right) \equiv q_{j}(a, b) \bmod p$, if $j<k$, and this implies that $f_{i}\left(a+d p^{k}, b\right) \equiv f_{i}(a, b) \bmod p$.

Now if $p$ does not divide $b$, then $h(a, b) \equiv 1 \bmod p$, and $q_{k}\left(a+d p^{k}, b\right) \equiv$ $q_{k}(a, b)+(-1)^{k-1} d b \bmod p$.

Therefore for all integers $d$

$$
\begin{aligned}
\sum_{i=0}^{p-1} f_{i}(a, b)\left(q_{k}(a, b)+(-1)^{k-1} d b\right)^{i} & \equiv f\left(a+d p^{k}, b\right) \bmod p \\
& \equiv 0 \bmod p,
\end{aligned}
$$

implying that $f_{i}(a, b) \equiv 0 \bmod p$, for $i=0,1, \ldots, p-1$.

If $p$ divides $b$, but not $a$, an almost identical argument, in which the roles of $a$ and $b$ are reversed, yields the same result.

Theorem 1.11. The polynomials $g_{m}^{n}(x, y), m=0,1, \ldots, n$, provide a $p$-local basis for the group of homogeneous numerical polynomials of degree $n$.

Proof. Note first that the $g_{m}^{n}(x, y)$ are rationally independent. If this were not so there would be integers $\lambda_{0}, \lambda_{1}, \ldots, \lambda_{n}$, not all divisible by $p$, such that $\sum_{m=0}^{n} \lambda_{m} g_{m}^{n}(x, y)=0$, which would contradict the lemma.

The group of homogeneous polynomials of degree $n$ has rank $n+1$ so that $g_{0}^{n}(x, y), g_{1}^{n}(x, y), \ldots, g_{n}^{n}(x, y)$ form a rational basis.

Given a homogeneous numerical polynomial $f(x, y)$ of degree $n$, let $f(x, y)$ $=\sum_{m=0}^{n} \mu_{m} g_{m}^{n}(x, y)$ with $\mu_{m} \in \mathbf{Q}$. Choose the integer $N$ so that $N \mu_{m} \in \mathbf{Z}$ for $m=0,1, \ldots, n$, and $p$ does not divide $N \mu_{j}$ for some $j$. If $p$ divided $N$ then the lemma would be contradicted. Hence $\mu_{m}$ is a $p$-local integer for all $m$.

In [47] one of us has considered certain algebraic consequences of the existence of this basis.

\section{THE $K$-THEORY OF $B U(n)$}

If we now think of $T^{n}$ as the maximal torus of the unitary group $U(n)$, the map $B T^{n} \rightarrow B U(n)$ induces an epimorphism $A_{n}=K_{0}\left(B T^{n}\right) \rightarrow K_{0}(B U(n))$ under which $\left(\begin{array}{c}w_{i} \\ k\end{array}\right)$ is mapped to $\beta_{k}$, for all $i$, so that $\left(\begin{array}{c}w_{1} \\ k_{1}\end{array}\right)\left(\begin{array}{c}w_{1} \\ k_{2}\end{array}\right) \cdots\left(\begin{array}{c}w_{n} \\ k_{n}\end{array}\right)$ is mapped to $\beta_{k_{1}} \beta_{k_{2}} \ldots \beta_{k_{n}}$.

Recall that $K_{0}(B U(n))$ has a basis (as a Z-module) consisting of the monomials in the $\beta_{k}$ of length at most $n$ [4]. Note that these monomials are with 
respect to the product induced by the Whitney sum, while the product which we have been using in $K_{0}\left(B T^{n}\right)$ comes from the tensor product of bundles.

We can, however, give a different description of $K_{0}(B U(n))$ in terms of polynomials satisfying an integrality condition.

Theorem 2.1. As a $K_{0}(K)$-comodule $K_{0}(B U(n))$ may be identified with the subcomodule of $\mathbf{Q}\left[x_{1}, \ldots, x_{n}\right]$ consisting of those symmetric polynomials $f\left(x_{1}, \ldots, x_{n}\right)$ satisfying

$$
\frac{n !}{n_{1} ! \cdots n_{r} !} f\left(k_{1}, \ldots, k_{n}\right) \in \mathbf{Z},
$$

where the sequence of integers $k_{1}, \ldots, k_{n}$ contains $r$ distinct elements repeated $n_{1}, \ldots, n_{r}$ times, respectively. Here $\mathbf{Q}\left[x_{1}, \ldots, x_{n}\right]$ has the multiplicative comodule structure given by $\psi\left(x_{i}\right)=w \otimes x_{i}$.

The map $A_{n}=K_{0}\left(B T^{n}\right) \rightarrow K_{0}(B U(n))$ sends a numerical polynomial $f\left(w_{1}, \ldots, w_{n}\right)$ to the symmetrization $(1 / n !) \sum_{\sigma \in S_{n}} f\left(x_{\sigma(1)}, \ldots, x_{\sigma(n)}\right)$.

Proof. This is a special case of a result in the appendix of [39], which provides a description of $K_{0}(B G)$ as a $K_{0}(K)$-comodule for any compact, connected Lie group $G$. An explicit proof of the case in question may be found in [47]. For an explanation of the significance of the variables $x_{i}$ see [43].

Corollary 2.2. The group of primitive elements $P_{m} K_{0}(B U(n))$ may be identified with the group of homogeneous symmetric polynomials of degree $m$ satisfying the integrality condition of the theorem.

Though $j_{*}: K_{0}\left(B T^{n}\right) \rightarrow K_{0}(B U(n))$ is, of course, onto, the restriction of $j_{*}$ to the graded group of primitive elements is not an epimorphism, as the following example shows.

Example 2.3. Let $g_{r}\left(x_{1}, x_{2}\right)=\frac{1}{8} x_{1}^{r} x_{2}^{r}\left(x_{1}-x_{2}\right)^{2}$, which is clearly symmetric and homogeneous. It is trivial to verify that if $r \geq 2$ then $g_{r}\left(x_{1}, x_{2}\right)$ satisfies the integrality conditions of Theorem 2.1. Hence $g_{r}\left(x_{1}, x_{2}\right) \in P_{2 r+2} K_{0}(B U(2))$ for $r \geq 2$.

Let $f_{r}\left(w_{1}, w_{2}\right)=\frac{1}{4} w_{1}^{r} w_{2}^{r}\left(w_{1}-w_{2}\right)\left(w_{1}-1\right)$; then $f_{r}\left(w_{1}, w_{2}\right) \in K_{0}\left(B T^{2}\right)$ for $r \geq 2$, and

$$
\begin{aligned}
j_{*}\left(f_{r}\left(w_{1}, w_{2}\right)\right) & =\frac{1}{2}\left(f_{r}\left(x_{1}, x_{2}\right)+f_{r}\left(x_{2}, x_{1}\right)\right) \\
& =\frac{1}{8} x_{1}^{r} x_{2}^{r}\left(\left(x_{1}-x_{2}\right)\left(x_{1}-1\right)+\left(x_{2}-x_{1}\right)\left(x_{2}-1\right)\right) \\
& =g_{r}\left(x_{1}, x_{2}\right) .
\end{aligned}
$$

However $f_{r}\left(w_{1}, w_{2}\right)$ is not homogeneous, i.e., not primitive. We will show that $g_{r}\left(x_{1}, x_{2}\right)$ does not belong to the image of

$$
j_{*}: P_{2 r+2} K_{0}\left(B T^{2}\right) \rightarrow P_{2 r+2} K_{0}(B U(2)) .
$$

Assume that $j_{*}$ maps $h_{r}\left(w_{1}, w_{2}\right) \in P_{2 r+2} K_{0}\left(B T^{2}\right)$ to $g_{r}\left(x_{1}, x_{2}\right)$. Thus

$$
\frac{1}{2}\left(h_{r}\left(x_{1}, x_{2}\right)+h_{r}\left(x_{2}, x_{1}\right)\right)=g_{r}\left(x_{1}, x_{2}\right) \text {, }
$$


so that $h_{r}(x, x)=g_{r}(x, x)=0$. Therefore $x_{1}-x_{2}$ divides $h_{r}\left(x_{1}, x_{2}\right)$, in $\mathbf{Q}\left[x_{1}, x_{2}\right]$.

Write $h_{r}\left(x_{1}, x_{2}\right)=\frac{1}{4}\left(x_{1}-x_{2}\right) s_{r}\left(x_{1}, x_{2}\right)$; then

$$
s_{r}\left(x_{1}, x_{2}\right)-s_{r}\left(x_{2}, x_{1}\right)=x_{1}^{r} x_{2}^{r}\left(x_{1}-x_{2}\right) \text {. }
$$

Since $h_{r}\left(x_{1}, x_{2}\right)$ is homogeneous of degree $2 r+2$, it follows that $s_{r}\left(x_{1}, x_{2}\right)$ is homogeneous of degree $2 r+1$, and thus that $s_{r}(x,-x)=-s_{r}(-x, x)$.

Thus, setting $x_{1}=x, x_{2}=-x$ in the above relation, we obtain $s_{r}(x,-x)=$ $(-1)^{r} x^{2 r+1}$. Therefore $h_{r}(1,-1)=(-1)^{r} \frac{1}{2}$, contradicting the assumption that $h_{r}\left(w_{1}, w_{2}\right)$ was numerical.

We shall see in $\S 10$ that $g_{2}\left(x_{1}, x_{2}\right)$ does not belong to the image of the Hurewicz map $\pi_{12}^{S}(B U(2)) \rightarrow P_{6} K_{0}(B U(2))$.

We conclude this section by establishing notation for some standard primitive elements and giving various formulas which relate them.

Definition 2.4. Let $\Sigma_{n} \in \pi_{2 n}(B U)$ denote the Bott generator and also its image in $\pi_{2 n}^{S}(B U)$ and $P_{n} K_{0}(B U)$.

Being unstably spherical, $\Sigma_{n}$ is also primitive in another sense. Since $K_{0}(B U)$ is torsion-free it possesses a diagonal map

$$
\Delta: K_{0}(B U) \rightarrow K_{0}(B U) \otimes K_{0}(B U),
$$

which makes it a coalgebra (in fact a Hopf algebra with respect to Whitney sum). An element $\alpha \in K_{0}(B U)$ is diagonal primitive if

$$
\Delta(\alpha)=\alpha \otimes 1+1 \otimes \alpha .
$$

The primitive elements which are our main object of study will be referred to as coaction primitives if the context is not clear.

It follows immediately from the definition that $B_{*}\left(\Sigma_{n}\right)=\Sigma_{n+1}$, under the Bott map $B_{*}: K_{0}(B U) \rightarrow K_{0}(B U)$.

Now $\Sigma_{1}=\beta_{1}$ and, accordingly to [5],

$$
B_{*}\left(\beta_{n}\right)=(-1)^{n}\left(\sigma_{n+1}-\sigma_{n}\right),
$$

where $\sigma_{i}$ is the Newton polynomial $N_{i}\left(\beta_{1}, \ldots, \beta_{i}\right)$ in $\beta_{1}, \ldots, \beta_{i}$.

Since $\sigma_{i} \equiv(-1)^{i-1} i \beta_{i}$ modulo decomposables, and $B_{*}$ annihilates decomposables,

$$
B_{*}\left(\sigma_{n}\right)=n\left(\sigma_{n}-\sigma_{n+1}\right) .
$$

Thus

$$
\begin{aligned}
\Sigma_{2} & =B_{*}\left(\Sigma_{1}\right)=\sigma_{1}-\sigma_{2}=\beta_{1}+2 \beta_{2}-\beta_{1}^{2}, \\
\Sigma_{3} & =B_{*}\left(\Sigma_{2}\right)=\sigma_{1}-3 \sigma_{2}+2 \sigma_{3} \\
& =\beta_{1}+6 \beta_{2}+6 \beta_{3}-3 \beta_{1}^{2}-6 \beta_{1} \beta_{2}+2 \beta_{1}^{3},
\end{aligned}
$$


and in general

$$
\Sigma_{k}=\sum_{i=1}^{k}(-1)^{i-1}(i-1) ! S_{k}^{i} \sigma_{i} .
$$

Here $S_{k}^{i}$ is the Stirling number of the second kind [11], defined by the identity

$$
w^{k}=\sum_{i=1}^{k} i ! S_{k}^{i}\left(\begin{array}{c}
w \\
i
\end{array}\right) .
$$

The elements $\Sigma_{n} \in P_{n} K_{0}(B U)$ will provide the building blocks out of which divisiblity relations will be constructed. They are, of course, geometrically very natural. However their expression in terms of the algebra with which we have been analyzing the primitives of $K_{0}(B U)$ is rather complicated.

We shall therefore need another basic set of elements, which like the $\Sigma_{n}$ are rational polynomial generators for $P_{*} K_{0}(B U)$, but which are algebraically simpler.

Let the image of $w^{k} \in P_{k} K_{0}\left(C P^{\infty}\right)$ in $P_{k} K_{0}(B U)$ be denoted by $\Gamma_{k}$. Since $\sigma_{i} \equiv(-1)^{i-1} i \beta_{i}$ modulo decomposables and $\beta_{i}=\left(\begin{array}{c}w \\ i\end{array}\right)$ in $K_{0}\left(C P^{\infty}\right)$, comparison of the formula for $\Sigma_{k}$ in terms of the $\sigma_{i}$ with the formula (2.6) which defines the $S_{k}^{i}$ shows that $\Sigma_{k} \equiv \Gamma_{k}$ modulo decomposables in $K_{0}(B U)$. In fact the two are congruent modulo decomposables in $P_{*} K_{0}(B U)$.

It follows that, for $k>0$,

$$
B_{*}\left(\Gamma_{k}\right)=B_{*}\left(\Sigma_{k}\right)=\Sigma_{k+1} .
$$

Under the limit map $l: K_{0}(B U) \rightarrow K_{0}(K)=A\left[w^{-1}\right]$ of diagram (1.4), both $\Sigma_{k}$ and $\Gamma_{k}$ are sent to $w^{k}$. The image of $l$ is clearly the subgroup $A \subset A\left[w^{-1}\right]$; in fact $l$ induces an isomorphism between the indecomposable module $Q K_{0}(B U)$ and the subgroup of $A$ consisting of the numerical polynomials without constant term. The group $\Delta K_{0}(B U)$ of diagonal primitive elements of $K_{0}(B U)$, for which the $\sigma_{i}$ form an integral basis and the $\Sigma_{i}$ a rational basis, maps monomorphically into $Q K_{0}(B U)$. Since $\sigma_{i} \equiv(-1)^{i-1} i \beta_{i}$ modulo decomposables, $l\left(\sigma_{i}\right)=(-1)^{i-1} i\left(\begin{array}{c}w \\ i\end{array}\right)$. It follows that $l$ maps $\Delta K_{0}(B U)$ isomorphically onto $w A \subset A\left[w^{-1}\right]$. In other words we have proved the following result.

Proposition 2.8. If $f(w)=\sum_{i=0}^{r} \lambda_{i} w^{i} \in A$, there is a unique rational linear combination of the $\Sigma_{i}$ which $l$ maps to $w f(w)$. It is given by $\sum_{i=0}^{r} \lambda_{i} \Sigma_{i+1}$, which belongs to $K_{0}(B U)$ since it equals $B_{*} i_{*}(f(w)-f(0))+f(0) \Sigma_{1}$.

\section{The Algebra of $\lambda$-SEQUenCes AND $W$-POLYNOMIALS}

In this section we define the $W$-polynomials associated to a sequence of rational numbers $\lambda_{1}, \lambda_{2}, \ldots$ and prove the results on such polynomials which we shall need in $\S \S 4$ and 5 . There the $\lambda$-sequence will be the sequence 
$\lambda_{k}=(-1)^{k} B_{k} / k$, where $B_{k}$ is the Bernoulli number, and we shall prove that, subject to certain admissibility conditions, the associated $W$-polynomials are stably numerical.

Definition 3.1. Given a sequence $\lambda_{1}, \lambda_{2}, \ldots$ of rational numbers, define the additive homomorphism $\Lambda: \mathbf{Q}[w] \rightarrow \mathbf{Q}[w]$, by $\Lambda\left(w^{k}\right)=\lambda_{k}\left(w^{k}-1\right)$.

Definition 3.2. If $K=\left(k_{r}, \ldots, k_{1}\right)$ is a sequence of natural numbers we write len $(K)=r$ and $|K|=k_{r}+\cdots+k_{1}$. If $J=\left(j_{s}, \ldots, j_{1}\right)$ then $K J$ denotes the sequence $\left(k_{r}, \ldots, k_{1}, j_{s}, \ldots, j_{1}\right)$. In particular $K(k)=\left(k_{r}, \ldots, k_{1}, k\right)$, and $(k) K=\left(k, k_{r}, \ldots, k_{1}\right)$.

We let () denote the empty sequence.

If $k$ is a natural number and $K$ is nonempty we let $K+k$ denote the sequence $\left(k_{r}, \ldots, k_{2}, k_{1}+k\right)$.

Definition 3.3. Fix the $\lambda$-sequence $\lambda_{1}, \lambda_{2}, \ldots$. For each sequence $K$ define the $W$-polynomial $W_{K}(w) \in \mathbf{Q}[w]$ recursively by

$$
W_{()}(w)=1 \quad \text { and } \quad W_{(k) K}(w)=\Lambda\left(w^{k} W_{K}(w)\right) .
$$

Thus

$$
\begin{aligned}
W_{(k)}(w) & =\lambda_{k}\left(w^{k}-1\right), \\
W_{(j, k)}(w) & =\lambda_{k}\left(\lambda_{j+k}\left(w^{j+k}-1\right)-\lambda_{j}\left(w^{j}-1\right)\right),
\end{aligned}
$$

and so on.

Note that $W_{K}(w)$ has degree $|K|$.

Lemma 3.4. For any natural number $k$ and any nonempty sequence $K$,

$$
W_{K(k)}(w)=\lambda_{k}\left(W_{K+k}(w)-W_{K}(w)\right) .
$$

Proof. The result follows by induction on len $(K)$, using Definition 3.3.

Corollary 3.5. For all nonempty sequences $K$ we have $W_{K}(1)=0$.

The following multiplicative property of $W$-polynomials will be crucial in $\S \S 4$ and 5.

Theorem 3.6. In $\mathbf{Q}[w, z]$

$$
W_{K}(w z)=\sum_{I J=K} z^{|I|} W_{I}(w) W_{J}(z) .
$$

Proof. The proof is by induction on len $(K)$. If $K$ has length one the formula is trivial to verify.

It is convenient to write $z^{I}$ for $z^{|I|}$.

Consider $W_{K(k)}(w z)$ which, is equal to $\lambda_{k}\left(W_{K+k}(w z)-W_{K}(w z)\right)$, by the lemma. The lengths of both $K+k$ and $K$ are less than that of $K(k)$, so that we may use the induction hypothesis to expand

$$
W_{K(k)}(w z)=\lambda_{k}\left(\sum_{L M=K+k} z^{L} W_{L}(w) W_{M}(z)-\sum_{I J=K} z^{I} W_{I}(w) W_{J}(z)\right) .
$$


Now, in these two sums, $I$ and $L$ run through the same set of sequences, except when $I=K$ and $L=K+k$. Apart from these terms, if $I J=K$, $L=I$, and $L M=K+k$, then $M=J+k$.

Thus

$$
\begin{aligned}
W_{K(k)}(w z)= & \lambda_{k}\left(\sum_{\substack{I J=K \\
I \neq K}} z^{I} W_{I}(w)\left(W_{J+k}(z)-W_{J}(z)\right)\right. \\
& \left.+z^{K+k} W_{K+k}(w)-z^{K} W_{K}(w)\right) \\
= & \sum_{\substack{I J=K \\
I \neq K}} z^{I} W_{I}(w) W_{J(k)}(z)+\lambda_{k}\left(z^{K+k} W_{K+k}(w)-z^{K} W_{K}(w)\right),
\end{aligned}
$$

where we have used the lemma again to simplify each summand.

Now the final terms are

$$
\begin{aligned}
\lambda_{k}\left(z^{K+k} W_{K+k}(w)-z^{K} W_{K}(w)\right) \\
\quad=\lambda_{k} z^{K}\left(z^{k} W_{K+k}(w)-z^{k} W_{K}(w)+z^{k} W_{K}(w)-W_{K}(w)\right), \\
=z^{K+k} W_{K(k)}(w)+z^{K} W_{K}(w) W_{(k)}(z),
\end{aligned}
$$

using the lemma and the fact that $W_{(k)}(z)=\lambda_{k}\left(z^{k}-1\right)$.

This provides all the terms in the summation of the statement of the theorem.

\section{The KUMMER CONGRUENCES AND STABLy NUMERICAL POLYNOMIALS}

In this section we discuss various families of stably numerical polynomials. These elements of the $K$-theory cooperation algebra will lead, via the techniques of $\S 5$, to the primitives in the $K$-theory of $B U$ which will be realized in $\S 8$.

Certain cases of the elements which we construct are related to the number theory of $\S 11$ of [2]. We explain the connection with our results at the end of this section.

If $n$ is a nonzero, even integer and $p$ a prime, let $\nu_{p}(n)$ denote the exponent of $p$ in $n$. Thus $p^{\nu_{p}(n)}$ divides $n$ but $p^{1+\nu_{p}(n)}$ does not. Following Adams, we define $m(n)$ as that natural number for which

$$
\nu_{p}(m(n))= \begin{cases}0, & \text { if } p-1 \text { does not divide } n, \\ 1+\nu_{p}(n), & \text { if } p \text { is odd and } p-1 \text { divides } n, \\ 2+\nu_{2}(n), & \text { if } p=2 .\end{cases}
$$

It is a simple consequence of the structure of the group of units modulo a prime power that, for each nonzero, even $n$,

$$
\left(w^{n}-1\right) / m(n) \text { is stably numerical; }
$$

see Theorem 2.7 of [1]. 
The polynomials $\left(w^{n}-1\right) / m(n)$ are not divisible within the ring $A\left[w^{-1}\right]$, as can be seen by setting $w$ equal to a primitive $(p-1) p^{\nu_{p}(n)}$ th root of unity modulo $p^{1+\nu_{p}(n)}$ for each prime $p$ such that $p-1$ divides $n$. The argument must be slightly modified for $p=2$.

We wish to generalize this construction to obtain elements of $A\left[w^{-1}\right]$ involving a larger number of terms. Unfortunately, even though $A\left[w^{-1}\right]$ is known to be a free Z-module [6], we do not have a natural basis at our disposal.

Adams proved that $m(n)$ is the denominator of $B_{n} / 2 n$, where $B_{n}$ is the Bernoulli number, defined by the identity $t /\left(e^{t}-1\right)=\sum_{k>0} B_{k} t^{k} / k !$. See Theorem 2.6 of [1], but note the change of notation for the Bernoulli numbers. The earliest proof of this result appears to be in [51].

Thus, for each even $n$, the polynomial $\left(B_{n} / 2 n\right)\left(w^{n}-1\right)$ is stably numerical, being an integer multiple of $\left(w^{n}-1\right) / m(n)$. But the latter element can also be obtained from the former using the following result.

Proposition 4.2. If $f(w)$ and $(a / b) f(w)$ are both stably numerical, where a and $b$ are coprime integers, then $(1 / b) f(w)$ is stably numerical.

Proof. By the Euclidean algorithm there are integers $A$ and $B$ such that $1=$ $A a+B b$. Thus $(1 / b) f(w)=A(a / b) f(w)+B f(w) \in A\left[w^{-1}\right]$.

We will work with the elements $\left(B_{n} / 2 n\right)\left(w^{n}-1\right)$. Not only does this enable the number theory to be generalized, but also it turns out to be more natural geometrically. This is illustrated by the fact that the $e$-invariant of the image of a generator of $\pi_{2 n-1}(U)$ under the $J$-homomorphism is $\left(B_{n} / n\right)\left(w^{n}-1\right)$. See [2] or [52].

The family of numerical polynomials which we shall construct depends on a version of Kummer's congruences among the Bernoulli numbers.

Theorem 4.3 (Global Kummer congruences). The linear map

$$
\kappa: \mathbf{Q}[w] \rightarrow \mathbf{Q}[w]
$$

defined by

$$
\kappa\left(w^{k}\right)=(-1)^{k+1} \frac{B_{k+1}}{k+1}\left(w^{k+1}-1\right), \quad k \geq 0,
$$

maps the ring $A$ of numerical polynomials into the ring $A\left[w^{-1}\right]$ of stably $n u$ merical polynomials.

Proof. Define the linear map $\phi: A \rightarrow \mathbf{Q}[w]$ by

$$
\phi\left(\left(\begin{array}{l}
w \\
k
\end{array}\right)\right)=N_{k}\left(w^{-1}\left(\begin{array}{c}
w \\
2
\end{array}\right), \ldots, w^{-1}\left(\begin{array}{c}
w \\
k+1
\end{array}\right)\right), \quad k \geq 0,
$$

where $N_{k}$ is the $k$ th Newton polynomial, and we adopt the convention that $N_{0}=0$.

Note that $w^{-1}\left(\begin{array}{c}w \\ j\end{array}\right)=((w-1) \cdots(w-j+1)) / j$ ! is indeed a polynomial in $w$ which is clearly stably numerical. 
Since the binomial coefficient polynomials form a basis for $A$ and the Newton polynomials have integer coefficients, $\phi$ maps $A$ into $A\left[w^{-1}\right]$.

In fact, since $N_{k}$ has total degree $k$, we have $\left.w^{k} \phi\left(\begin{array}{c}w \\ k\end{array}\right)\right) \in A$ for each $k \geq 0$. Let the linear map $\beta: A \rightarrow A$ be defined by

$$
\beta\left(\left(\begin{array}{l}
w \\
k
\end{array}\right)\right)=(-1)^{k}\left(\left(\begin{array}{c}
w \\
k+1
\end{array}\right)-\left(\begin{array}{l}
w \\
k
\end{array}\right)\right), \quad k \geq 0 .
$$

We will show that $\kappa=\phi \circ \beta$, from which the result follows.

Let $F(t)=\sum_{k \geq 0}\left(\begin{array}{c}w \\ k\end{array}\right) t^{k}=(1+t)^{w} \in A[[t]]$, and let $F^{\phi \circ \beta}(t)$ denote the series in $A\left[w^{-1}\right][[t]]$ obtained by applying $\phi \circ \beta$ to the coefficients of $F(t)$. Thus

$$
\begin{aligned}
F^{\phi \circ \beta}(t) & =\sum_{k \geq 0}(-1)^{k}\left(N_{k+1}\left(w^{-1}\left(\begin{array}{c}
w \\
2
\end{array}\right), \ldots, w^{-1}\left(\begin{array}{c}
w \\
k+2
\end{array}\right)\right)\right. \\
& \left.-N_{k}\left(w^{-1}\left(\begin{array}{c}
w \\
2
\end{array}\right), \ldots, w^{-1}\left(\begin{array}{c}
w \\
k+1
\end{array}\right)\right)\right) t^{k} \\
& =(1+t) \sum_{k \geq 1}(-1)^{k-1} N_{k}\left(w^{-1}\left(\begin{array}{c}
w \\
2
\end{array}\right), \ldots, w^{-1}\left(\begin{array}{c}
w \\
k+1
\end{array}\right)\right) t^{k-1} \\
& =(1+t) \frac{G^{\prime}(t)}{G(t)}
\end{aligned}
$$

where $G(t)=\sum_{j \geq 0} w^{-1}\left(\begin{array}{c}w \\ j+1\end{array}\right) t^{j}$.

The last step follows from the identity

$$
\sum_{k \geq 1}(-1)^{k-1} N_{k}\left(c_{1}, \ldots, c_{k}\right) t^{k-1}=\frac{d}{d t} \log \left(\sum_{j \geq 0} c_{j} t^{j}\right),
$$

which could be taken as the definition of the Newton polynomials.

Now let $t=e^{s}-1=s+s^{2} / 2 !+s^{3} / 3 !+\cdots \in \mathbf{Q}[[s]]$; then $F\left(e^{s}-1\right)=e^{w s}=$ $\sum_{k \geq 0} w^{k} s^{k} / k$ ! , and $F^{\phi \circ \beta}\left(e^{s}-1\right)=e^{s} G^{\prime}\left(e^{s}-1\right) / G\left(e^{s}-1\right)$.

By the chain rule $G^{\prime}\left(e^{s}-1\right)=e^{-s}(d / d s) G\left(e^{s}-1\right)$, but from the power series definition of $G(t)$ we have

$$
G(t)=\left((1+t)^{w}-1\right) / w t
$$

so that

$$
G\left(e^{s}-1\right)=\left(e^{m s}-1\right) / w\left(e^{s}-1\right) .
$$

Thus

$$
\frac{d}{d s} G\left(e^{s}-1\right)=\frac{e^{w s}}{e^{s}-1}-\frac{\left(e^{w s}-1\right) e^{s}}{w\left(e^{s}-1\right)^{2}}
$$


so that

$$
\begin{aligned}
F^{\phi \circ \beta}\left(e^{s}-1\right) & =\frac{w e^{w s}}{e^{u s}-1}-\frac{e^{s}}{e^{s}-1} \\
& =w-1+\frac{w}{e^{w s}-1}-\frac{1}{e^{s}-1} \\
& =w-1+\sum_{k \geq 0} \frac{B_{k+1}}{k+1}\left(w^{k+1}-1\right) \frac{s^{k}}{k !} .
\end{aligned}
$$

Since $B_{1}=-\frac{1}{2}$ and the other odd Bernoulli numbers are zero, it follows that

$$
\phi \circ \beta\left(w^{k}\right)=(-1)^{k+1} \frac{B_{k+1}}{k+1}\left(w^{k+1}-1\right)=\kappa\left(w^{k}\right) \text {. }
$$

Our aim is to construct numerical polynomials with large denominators by iterating the map $\kappa$. However, since $\kappa$ transforms numerical polynomials into polynomials which are only stably numerical, each new application of $\kappa$ must be preceded by multiplication by a suitable power of $w$. The minimal allowable power is, in general, difficult to predict. The results of Johnson [27] imply that a general stably numerical polynomial of degree $m$ may have to be multiplied by $w^{2 m-\alpha(m)}$ before it becomes numerical, where $\alpha(m)$ denotes the sum of the dyadic digits of $m$. However the following proposition gives us a smaller, and simpler, upper bound for the case in which we are interested.

Proposition 4.4. Let $f(w) \in A$ have degree $n$; then $w^{n+1} \kappa(f(w)) \in A$.

Proof. Note that $\beta(f(w))$ is an integer linear combination of $1, w,\left(\begin{array}{l}w \\ 2\end{array}\right), \ldots$, $\left(\begin{array}{c}w \\ n+1\end{array}\right)$. Since $w^{k} \phi\left(\left(\begin{array}{l}w \\ k\end{array}\right)\right) \in A$ for all $k \geq 0$,

$$
w^{n+1} \kappa(f(w))=w^{n+1} \phi \circ \beta(f(w)) \in A .
$$

Proposition 4.5. If $k>0$ then $\left(B_{k} / k\right)\left(w^{k}-1\right) \in A\left[w^{-1}\right]$.

Proof. The given element is $(-1)^{k} \kappa\left(w^{k-1}\right)$.

If $k=1$ the proposition asserts that $(w-1) / 2$, which equals $w^{-1}\left(\begin{array}{l}w \\ 2\end{array}\right)$, is stably numerical. For other odd values of $k$ the result is vacuous.

If $k$ is even then the denominator of $B_{k} / k$ is $\frac{1}{2} m(k)$. Thus, apart from a factor of 2 , we have another construction of the elements of (4.1). The following result shows that the extra power of 2 can be recovered.

Proposition 4.6. If $k$ is even and positive then $\left(B_{k} / 2 k\right)\left(w^{k}-1\right) \in A\left[w^{-1}\right]$.

Proof. Apply the homomorphism $\kappa$ to $w^{k-1}(1-w) / 2 \in A$.

Since $k$ is even and positive, $B_{k+1}=0$ and we obtain the required element of $A\left[w^{-1}\right]$.

Proposition 4.7. If $j$ and $k$ are even and positive, with $j$ sufficiently large, then

$$
\frac{B_{k}}{2 k}\left(\frac{B_{j}}{2 j}\left(w^{j}-1\right)-\frac{B_{j+k}}{2 j+2 k}\left(w^{j+k}-1\right)\right) \in A\left[w^{-1}\right] .
$$


Proof. Apply $\kappa$ to

$$
\frac{w^{j-1}(1-w)}{2} \frac{B_{k}}{2 k}\left(w^{k}-1\right),
$$

which will be numerical if $j$ is greater than or equal to the maximum exponent of any prime in $2 m(k)$.

If $p$ is an odd prime then, since $p$ divides the denominator of $B_{p-1}$,

$$
\frac{1}{p}\left(\frac{B_{j}}{2 j}\left(w^{j}-1\right)-\frac{B_{j+p-1}}{2 j+2 p-2}\left(w^{j+p-1}-1\right)\right) \in A\left[w^{-1}\right] .
$$

If $p-1$ does not divide $j$, so that $B_{j} / 2 j$ is a $p$-local integer, we may set $w$ equal to a primitive $(p-1)$ th root of unity modulo $p$ to obtain the congruence

$$
B_{j} / 2 j \equiv B_{j+p-1} /(2 j+2 p-2) \bmod p .
$$

It is this result which is usually referred to as Kummer's congruence; see for example $\S 8$ of Chapter 5 of [13]. In fact the result in Kummer's [32] is slightly more general. It is the congruence obtained by applying this technique to the numerical polynomial $w^{i}\left(\left(w^{j+1}-w\right) / p\right)^{n}$, where $j$ is a multiple of $p-1$.

The process of constructing numerical polynomials may be continued. The next elements of $A\left[w^{-1}\right]$ which can be obtained by our method have the form

$$
\begin{aligned}
\frac{B_{k}}{2 k}\left(\frac{B_{j}}{2 j}\left(\frac{B_{i}}{2 i}\left(w^{i}-1\right)-\frac{B_{i+j}}{2 i+2 j}\left(w^{i+j}-1\right)\right)\right. & \\
& \left.-\frac{B_{j+k}}{2 j+2 k}\left(\frac{B_{i}}{2 i}\left(w^{i}-1\right)-\frac{B_{i+j+k}}{2 i+2 j+2 k}\left(w^{i+j+k}-1\right)\right)\right) .
\end{aligned}
$$

We state the general result in the language of $\S 3$.

Definition 4.8. A sequence $K=\left(k_{r}, \ldots, k_{1}\right)$ of positive integers is said to be admissible if $k_{j+1} \geq k_{j}+\cdots+k_{1}$ for $j=1,2, \ldots, r$. It is strictly admissible if the strict inequality holds for all relevant values of $j$.

Proposition 4.9. The $W$-polynomial $W_{K}(w)$ of Definition 3.3 corresponding to the $\lambda$-sequence $\lambda_{k}=(-1)^{k} B_{k} / k$ is stably numerical if the sequence $K$ is admissible. Moreover $w^{|K|} W_{K}(w)$ is numerical.

Proof. The homomorphism $\Lambda$ corresponding to this $\lambda$-sequence is characterized by $\Lambda(w f(w))=\kappa(f(w))$. Thus Theorem 4.3 and Proposition 4.4 imply that if $f(w) \in A$ has degree $n$ then $w^{k} \Lambda(f(w)) \in A$ if $k \geq n$. Since $W_{K}(w)$ has degree $|K|$ the result follows from Definition 3.3 by induction on $\operatorname{len}(K)$.

Corollary 4.10. If $K$ is admissible then $w^{2|K|} W_{K}\left(w^{-1}\right)$ is a numerical polynomial.

Proof. It follows directly from the proposition that $W_{K}\left(w^{-1}\right)$ is stably numerical and clearly $w^{|K|} W_{K}\left(w^{-1}\right)$ is a polynomial. The force of the corollary is 
that it becomes numerical if multiplied by $w^{|K|}$, which we prove by induction on $\operatorname{len}(K)$.

To start the induction note that $w^{2 k} W_{(k)}\left(w^{-1}\right)=-w^{k} W_{(k)}(w)$, since $W_{(k)}(w)=(-1)^{k}\left(B_{k} / k\right)\left(w^{k}-1\right)$.

In general set $z=w^{-1}$ in Theorem 3.6, and use Corollary 3.5 to obtain

$$
0=W_{K}(1)=\sum_{I J=K} w^{-|I|} W_{I}(w) W_{J}\left(w^{-1}\right) .
$$

Multiplying through by $w^{2|K|}$ and rearranging,

$$
w^{2|K|} W_{K}\left(w^{-1}\right)=-\sum_{\substack{I J \neq K \\ J \neq K}} w^{|I|} W_{I}(w) w^{2|J|} W_{J}\left(w^{-1}\right),
$$

since $2|K|-|I|=|I|+2|J|$ if $I J=K$.

Now by Proposition 4.9 and the induction hypothesis all the terms in this sum are numerical polynomials, and the proof is complete.

As we have seen in Proposition 4.7 for the case of sequences $K$ of length two, Proposition 4.9 remains true for the $\lambda$-sequence $\lambda_{k}=(-1)^{k} B_{k} / 2 k$, if $K$ is strictly admissible. However the elements which we shall realize in $\S 8$ are related to the $W$-polynomials formed without the extra 2 , as in the statement of Proposition 4.9.

If we work a prime at a time the admissibility conditions in the previous results can be eliminated. To do this we need the following local version of Theorem 4.3.

Theorem 4.11 (Local Kummer congruences). Let $p$ be a prime. The linear map

$$
\kappa_{p}: \mathbf{Q}[w] \rightarrow \mathbf{Q}[w]
$$

given by

$$
\kappa_{p}\left(w^{k}\right)=(-1)^{k+1}\left(1-p^{k}\right) \frac{B_{k+1}}{k+1}\left(w^{k+1}-1\right), \quad k \geq 0,
$$

maps the ring $A_{(p)}\left[w^{-1}\right] \cap \mathbf{Q}[w]$ of p-local stably numerical polynomials into itself.

Proof. See the introduction of [28], where Katz states an equivalent result which follows from the existence of the $p$-adic Bernoulli measures.

We have not been able to find an elementary proof of Theorem 4.11 along the lines of the proof of Theorem 4.3. The most accessible account of the measure theoretic approach is to be found in [30].

Katz remarks that these congruences were "essentially known to Kummer." However the extra factors $\left(1-p^{k}\right)$ do not, as far as we know, occur in Kummer's work. The full congruences do not seem to have been noticed until the work of Kubota and Leopoldt [31] on $p$-adic zeta functions.

Notice that the difficulties in iterating the map $\kappa$ of Theorem 4.3, which we discussed after the proof of that theorem, do not arise for the local $\kappa_{p}$. 
Corollary 4.12. If $K=\left(k_{r}, \ldots, k_{1}\right)$ is a sequence of natural numbers then the polynomial $W_{K}(w)$ corresponding to the $\lambda$-sequence

$$
\lambda_{k}=(-1)^{k+1}\left(1-p^{k}\right) B_{k+1} /(k+1)
$$

is a p-local stably numerical polynomial.

The trinomial case of these polynomials, which are local versions of the polynomials of Proposition 4.7, occur, at least implicitly, in [2]. There Adams shows, in Lemma 11.2, that for $u$ and $v$ distinct, nonzero, even integers, there exists a rational number $\delta(u, v)$ such that for sufficiently large $t$ and for all integers $n$

$$
\left(n^{t+u}-n^{t}\right)-\delta(u, v)\left(n^{t+v}-n^{t}\right) \equiv 0 \bmod m(u) m(v-u) .
$$

We now show how our results give simple congruences for the numbers $\delta(u, v)$, which differ from those in [2]. It is the presence of the Bernoulli numbers in our formulas which systematizes our approach and enables the results to be generalized.

Adams' result can be restated in terms of numerical polynomials as the fact that, if $j$ and $k$ are even,

$$
\frac{1}{m(k)}\left(\frac{w^{j}-1}{m(j)}-\gamma(j, k) \frac{w^{j+k}-1}{m(j+k)}\right) \in A\left[w^{-1}\right],
$$

where $\gamma(j, k)$ is the integer $m(k) \delta(j, j+k)$.

Comparing this with the polynomials $W_{(j, k)}(w)$ associated to the $\lambda$-sequence $\lambda_{k}=(-1)^{k+1}\left(1-p^{k}\right) B_{k+1} /(2 k+2)$, we obtain the congruence

$$
\left(1-p^{j-1}\right) n(j) \gamma(j, k) \equiv n(j+k) \quad \bmod p^{n+1},
$$

for all odd primes $p$ such that $p^{n}(p-1)$ divides $k$, where $n(j)$ denotes the numerator of $B_{j} / j$. If $p=2$ and $2^{n}$ divides $k$, the congruence (4.13) holds modulo $2^{n+2}$.

If $j$ sufficiently large we have the global congruence

$$
n(j) \gamma(j, k) \equiv n(j+k) \quad \bmod m(k) .
$$

The congruences 4.13 determine $\gamma(j, k)$, as long as none of the primes $p$ such that $p-1$ divides $k$ also divide $n(j)$. However in this case, which corresponds to part (i) of the proof of 11.2 in [2], $\gamma(j, k)$ is simply chosen modulo $p^{n+1}$ so that the constant terms cancel modulo $p^{n+1}$ in

$$
\left(w^{j}-1\right) / m(j)-\gamma(j, k)\left(w^{j+k}-1\right) / m(j+k),
$$

and thus

$$
\frac{1}{m(k)}\left(\frac{w^{j}-1}{m(j)}-\gamma(j, k) \frac{w^{j+k}-1}{m(j+k)}\right) \equiv w^{j}\left(\frac{w^{k}-1}{m(k)}\right) \quad \bmod \mathbf{Z}_{(p)}[w] .
$$

Hence Adams' approach does not generate any more numerical polynomials than ours. 
Murdock has used Adams' function $\delta(u, v)$ to study the $e$-invariant on twocell complexes [38]. He states general integrality conditions for the values of the $e$-invariant in an $n$-cell complex, in terms of numerical polynomials, but remarks that "the functions playing the role of $\delta(u, v)$ seem to be unknown." They are essentially provided by the existence of the elements of the $K$-theory cooperation algebra given by Corollary 4.12 .

\section{Constructing PRimitive elements IN the $K$-THeORy OF $B U$}

In this section we investigate techniques for obtaining elements in the groups $P_{m} K_{0}(B U(n))$. In the light of Theorem 2.1 this means that we need to be able to construct homogeneous symmetric polynomials satisfying a certain integrality condition. The families which we are able to construct derive initially from a two-dimensional torus and thus from homogeneous numerical polynomials in two variables.

We will write our primitive elements as rational polynomials in the elements $\Sigma_{i}$ or $\Gamma_{i}$. Application of the Chern character shows that $P_{*} K_{0}(B U) \otimes \mathbf{Q}$ is a polynomial algebra generated by $\Sigma_{1}, \Sigma_{2}, \ldots$. The $\Gamma_{i}$ will also serve as polynomial generators. No power of the $\Sigma_{i}$ or of the $\Gamma_{i}$ is divisible, but more complicated expressions may well be.

For example $\Gamma_{1} \Gamma_{3}-\Gamma_{2}^{2} \in P_{4} K_{0}(B U)$ comes from

$$
w_{1} w_{2}^{3}-w_{1}^{2} w_{2}^{2}=w_{1} w_{2}^{2}\left(w_{2}-w_{1}\right)
$$

in $P_{4} K_{0}\left(B T^{2}\right)$. This element is clearly divisible by 2 in $K_{0}\left(B T^{2}\right) \cong A_{2}$. Hence $\frac{1}{2}\left(\Gamma_{1} \Gamma_{3}-\Gamma_{2}^{2}\right) \in P_{4} K_{0}(B U)$.

Constructing primitive elements in this fashion leads to polynomial expressions in the $\Gamma_{i}$. We now explain how to obtain elements involving the $\Sigma_{i}$.

If we think of $B T^{2}$ as $C P^{\infty} \times C P^{\infty}$ and map each $C P^{\infty}$ separately into a copy of $B U$, the polynomial $\frac{1}{2} w_{1} w_{2}^{2}\left(w_{2}-w_{1}\right)$ gives rise to $\frac{1}{2}\left(\Gamma_{1} \otimes \Gamma_{3}-\Gamma_{2} \otimes \Gamma_{2}\right)$ in $K_{0}(B U \times B U) \cong K_{0}(B U) \otimes K_{0}(B U)$. Applying the Bott map to each factor and using formula (2.7), we obtain $\frac{1}{2}\left(\Sigma_{2} \otimes \Sigma_{4}-\Sigma_{3} \otimes \Sigma_{3}\right)$, so that, applying the Whitney sum, $\frac{1}{2}\left(\Sigma_{2} \Sigma_{4}-\Sigma_{3}^{2}\right) \in P_{6} K_{0}(B U)$. This element is primitive since each monomial has weight 6 .

For the general case let $f\left(w_{1}, w_{2}\right) \in A_{2}$ be homogeneous of degree $r$. We may assume that $f(w, 0)=f(0, w)=0$, since otherwise we can replace $f\left(w_{1}, w_{2}\right)$ by $f\left(w_{1}, w_{2}\right)-f(1,0) w_{1}^{r}-f(0,1) w_{2}^{r}$.

If $f\left(w_{1}, w_{2}\right)=\sum_{j=1}^{r-1} \lambda_{j} w_{1}^{j} w_{2}^{r-j}$, with $\lambda_{j} \in \mathbf{Q}$, then we obtain

$$
\sum_{j=1}^{r-1} \lambda_{j} \Gamma_{j} \Gamma_{r-j} \in P_{r} K_{0}(B U),
$$

and

$$
\sum_{j=1}^{r-1} \lambda_{j} \Sigma_{j+1} \Sigma_{r-j+1} \in P_{r+2} K_{0}(B U) .
$$


Using the Bott map on only one factor gives rise to a third type of primitive:

$$
\sum_{j=1}^{r-1} \lambda_{j} \Gamma_{j} \Sigma_{r-j+1} \in P_{r+1} K_{0}(B U) .
$$

Elements of the form (5.2) may be identified in another way. The element $\sum_{j=1}^{r-1} \lambda_{j} \Sigma_{j+1} \otimes \Sigma_{r-j+1} \in K_{0}(B U \times B U)$ is mapped by

$$
l \otimes l: K_{0}(B U \times B U) \rightarrow K_{0}(K) \otimes K_{0}(K)
$$

to $w_{1} w_{2} f\left(w_{1}, w_{2}\right)$ in $w_{1} w_{2} A_{2} \cong w A \otimes w A \subset A\left[w^{-1}\right] \otimes A\left[w^{-1}\right]$, where $w_{1}=$ $w \otimes 1$ and $w_{2}=1 \otimes w$. Since $l$ is a monomorphism when restricted to the summand rationally generated by the $\Sigma_{i}$, the element $\sum_{j=1}^{r-1} \lambda_{j} \Sigma_{j+1} \otimes \Sigma_{r-j+1}$ may be characterized as the unique rational linear combination of the $\Sigma_{i} \otimes \Sigma_{j}$ which is mapped to $w_{1} w_{2} f\left(w_{1}, w_{2}\right)$ by $l \otimes l$.

Similarly, $\sum_{j=1}^{r-1} \lambda_{j} w^{j} \otimes \Sigma_{r-j+1} \in K_{0}\left(C P^{\infty} \times B U\right) \cong A \otimes K_{0}(B U)$ is the unique rational linear combination of the terms $w^{i} \otimes \Sigma_{j}$ which is mapped to $w_{2} f\left(w_{1}, w_{2}\right) \in A \otimes w A \subset A \otimes A\left[w^{-1}\right] \cong K_{0}\left(C P^{\infty}\right) \otimes K_{0}(K)$ by $1 \otimes l$.

In $\S 9$ we will construct and realize some primitives of the form (5.3). We now use the results of $\S 4$ to generate a family of primitives of the form (5.2) which will be realized in $\S 8$.

If the sequence $K=\left(k_{r}, \ldots, k_{1}\right)$ is admissible and $j>|K|$, then, by Proposition 4.9 and Corollary $4.10, w^{j} W_{K}(w)$ and $w^{j+|K|} W_{K}\left(w^{-1}\right)$ belong to $w A$. Definition 5.4. For such $j$ and $K$ we let $\Delta_{j, K}$ and $\nabla_{j, K}$ be the unique diagonal primitive elements of $K_{0}(B U)$ which map under $l: K_{0}(B U) \rightarrow K_{0}(K)$ to $w^{j} W_{K}(w)$ and $w^{j+|K|} W_{K}\left(w^{-1}\right)$, respectively.

The existence and uniqueness of these elements are guaranteed by Proposition 2.8 .

Thus $\Delta_{j,()}=\nabla_{j,()}=\Sigma_{j}$, and $\Delta_{j,(k)}=-\nabla_{j,(k)}=(-1)^{k}\left(B_{k} / k\right)\left(\Sigma_{j+k}-\Sigma_{j}\right)$.

Proposition 5.5. $\Delta_{j, K}$ and $\nabla_{j, K}$ may be defined recursively by

$$
\begin{gathered}
\Delta_{j, K(k)}=(-1)^{k}\left(B_{k} / k\right)\left(\Delta_{j, K+k}-\Delta_{j, K}\right), \\
\nabla_{j, K(k)}=(-1)^{k}\left(B_{k} / k\right)\left(\nabla_{j, K+k}-\nabla_{j+k, K}\right),
\end{gathered}
$$

for every nonempty sequence $K$.

Proof. This follows immediately from Lemma 3.4.

If we now set $w=w_{1}$ and $z=w_{2}^{-1}$ in Theorem 3.6,

$$
W_{K}\left(w_{1} w_{2}^{-1}\right)=\sum_{I J=K} w_{2}^{-|I|} W_{I}\left(w_{1}\right) W_{J}\left(w_{2}^{-1}\right) .
$$

Multiplying by $w_{1}^{|K|} w_{2}^{2|K|}$,

$$
w_{1}^{|K|} w_{2}^{2|K|} W_{K}\left(w_{1} w_{2}^{-1}\right)=\sum_{I J=K} w_{1}^{|J|} w_{2}^{2|I|} w_{1}^{|I|} W_{I}\left(w_{1}\right) w_{2}^{2|J|} W_{J}\left(w_{2}^{-1}\right),
$$


which by Proposition 4.9 and Corollary 4.10 belongs to $A_{2}$, the ring of numerical polynomials in two variables.

Note that $w_{1}^{i} w_{2}^{j} W_{K}\left(w_{1} w_{2}^{-1}\right)$ is clearly numerical for sufficiently large $i$ and $j$ since $W_{K}(w)$ is stably numerical; see Proposition 1.9. The advantage of using Theorem 3.6 is to provide a specific bound on such $i$ and $j$.

If $i, j>|K|$ the element

$$
w_{1}^{i} w_{2}^{j+|K|} W_{K}\left(w_{1} w_{2}^{-1}\right)=\sum_{I J=K} w_{1}^{i} W_{I}\left(w_{1}\right) w_{2}^{j+|J|} W_{J}\left(w_{2}^{-1}\right)
$$

can be lifted to a unique rational linear combination of the elements $\Sigma_{i} \otimes \Sigma_{j}$ in $K_{0}(B U \times B U)$.

Definition 5.7. Let $\Xi_{i, j, K} \in P_{i+j+|K|} K_{0}(B U)$ denote the image of this linear combination under the Whitney sum map.

Thus $\Xi_{i, j, K}$ is the element of the form (5.2) corresponding to the homogeneous numerical polynomial $w_{1}^{i-1} w_{2}^{j-1} W_{K}\left(w_{1} w_{2}^{-1}\right)$.

Proposition 5.8. If $K$ is a nonempty sequence,

$$
\Xi_{i, j, K(k)}=(-1)^{k}\left(B_{k} / k\right)\left(\Xi_{i, j, K+k}-\Xi_{i, j+k, K}\right),
$$

which may be used to define the $\Xi_{i, j, K}$ recursively.

Proof. This follows from Lemma 3.4.

For example,

$$
\begin{aligned}
\Xi_{i, j,()} & =\Sigma_{i} \Sigma_{j}, \\
\Xi_{i, j,(a)} & =(-1)^{a} \frac{B_{a}}{a}\left(\Sigma_{i+a} \Sigma_{j}-\Sigma_{i} \Sigma_{j+a}\right), \\
\Xi_{i, j,(a, b)} & =(-1)^{b} \frac{B_{b}}{b}\left(\Xi_{i, j,(a+b)}-\Xi_{i, j+b,(a)}\right) \\
& =(-1)^{b} \frac{B_{b}}{b}\left((-1)^{a+b} \frac{B_{a+b}}{a+b}\left(\Sigma_{i+a+b} \Sigma_{j}-\Sigma_{i} \Sigma_{j+a+b}\right)\right. \\
& \left.\quad-(-1)^{a} \frac{B_{a}}{a}\left(\Sigma_{i+a} \Sigma_{j+b}-\Sigma_{i} \Sigma_{j+a+b}\right)\right) .
\end{aligned}
$$

Theorem 5.9. The following equation holds in $K_{0}(B U)$ :

$$
\Xi_{i, j, K}=\sum_{I J=K} \Delta_{i, I} \nabla_{j, J} .
$$

Proof. This is an immediate consequence of the formula (5.6).

This fundamental formula is the one which we shall use to realize $\Xi_{i, j, K}$ geometrically. It would not, however, be convenient to define $\Xi_{i, j, K}$ in this way, for a large amount of cancellation takes place. It is not even obvious that the right-hand side is a weighted homogeneous expression in the $\Sigma_{i}$, which is the condition for it to be primitive. 
One must not be misled by the obvious consequence of Theorem 5.9 that the $\Xi_{i, j, K}$ are decomposable in the ring $K_{0}(B U)$ into believing that this is the case in the graded ring $P_{*} K_{0}(B U)$.

\section{THE $K$-THEORY OF ITERATED THOM COMPLEXES}

The algebra which we used in $\S 4$ to prove various cases of the Kummer congruences is closely related to the Thom isomorphism in $K$-theory. The following result shows this most clearly and leads us to a fuller understanding of the $\Delta$-elements of Definition 5.4.

We should remark that in the proof of Proposition 1.3 we have already chosen the reduced Hopf bundle as a preferred power series generator for $K^{0}\left(C P^{\infty}\right)$. Following [4], this leads to a choice of Thom class $t: M U \rightarrow K$, which agrees with that of [52].

Proposition 6.1. The following diagram commutes:

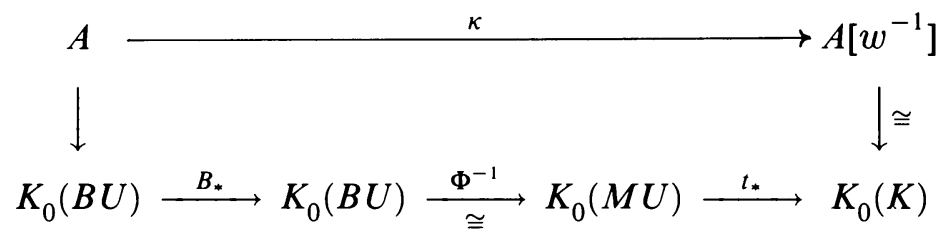

where $\kappa$ is the homomorphism defined in Theorem 4.3, $B_{*}$ is the Bott map, $t: M U \rightarrow K$ is the $K$-theory Thom class, and $\Phi$ the corresponding Thom isomorphism.

Proof. In the notation of the proof of Theorem 4.3,

$$
\begin{aligned}
\kappa\left(\left(\begin{array}{l}
w \\
k
\end{array}\right)\right) & =\phi \circ \beta\left(\left(\begin{array}{l}
w \\
k
\end{array}\right)\right) \\
& =\phi\left((-1)^{k}\left(\left(\begin{array}{c}
w \\
k+1
\end{array}\right)-\left(\begin{array}{l}
w \\
k
\end{array}\right)\right)\right) \\
= & (-1)^{k}\left(N_{k+1}\left(w^{-1}\left(\begin{array}{l}
w \\
2
\end{array}\right), \ldots, w^{-1}\left(\begin{array}{c}
w \\
k+2
\end{array}\right)\right)\right. \\
& \left.\quad-N_{k}\left(w^{-1}\left(\begin{array}{l}
w \\
2
\end{array}\right), \ldots, w^{-1}\left(\begin{array}{c}
w \\
k+1
\end{array}\right)\right)\right) .
\end{aligned}
$$

On the other hand $\left(\begin{array}{c}w \\ k\end{array}\right) \in A$ maps to $\beta_{k} \in K_{0}(B U)$ and

$$
B_{*}\left(\beta_{k}\right)=(-1)^{k}\left(N_{k+1}\left(\beta_{1}, \ldots, \beta_{k+1}\right)-N_{k}\left(\beta_{1}, \ldots, \beta_{k}\right)\right) ;
$$

see equation (2.5).

Now $\Phi$ and $t_{*}$ are both ring homomorphisms, while $\Phi\left(b_{i}^{K}\right)=\beta_{i}$ and $t_{*}\left(b_{i}^{K}\right)=w^{-1}\left(\begin{array}{c}w \\ i+1\end{array}\right)$; see pages 400 and 435 of [52]: So

$$
\begin{aligned}
& t_{*} \Phi^{-1} B_{*}\left(\beta_{k}\right)=(-1)^{k}\left(N_{k+1}\left(w^{-1}\left(\begin{array}{c}
w \\
2
\end{array}\right), \ldots, w^{-1}\left(\begin{array}{c}
w \\
k+2
\end{array}\right)\right)\right. \\
&-\left.N_{k}\left(w^{-1}\left(\begin{array}{c}
w \\
2
\end{array}\right), \ldots, w^{-1}\left(\begin{array}{c}
w \\
k+1
\end{array}\right)\right)\right),
\end{aligned}
$$

and the diagram commutes. 
If a different $K$-theory orientation class is used to define both the Thom isomorphism $\Phi$ and the Thom class $t: M U \rightarrow K$ then the diagram fails to commute only by a function $A \rightarrow \mathbf{Z}[w]$. Thus no new stably numerical polynomials arise.

\section{Corollary 6.2.}

$$
t_{*} \Phi^{-1}\left(\Sigma_{k}\right)=(-1)^{k}\left(B_{k} / k\right)\left(w^{k}-1\right), \quad k \geq 1 .
$$

Proof. $w^{k-1} \in A$ maps to $\Gamma_{k-1}$ in $K_{0}(B U)$ and $B_{*}\left(\Gamma_{k-1}\right)=\Sigma_{k}$, by (2.7), while $\kappa\left(w^{k-1}\right)=(-1)^{k}\left(B_{k} / k\right)\left(w^{k}-1\right)$.

Our next step is an unstable version of this corollary. If $k \leq j$ then $\Sigma_{k}$ belongs to $K_{0}(B U(j)) \subset K_{0}(B U)$.

The Thom class $t \in \widetilde{K}^{0}(M U(j))$ may be represented by a map

$$
t: M U(j) \rightarrow B U,
$$

and the diagram

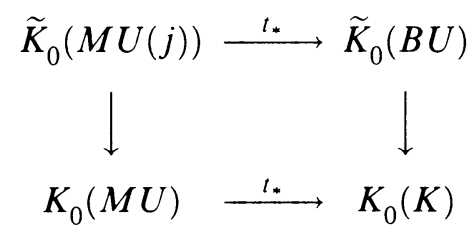

commutes, where $K_{0}(B U)$ must be interpreted as the $j$ th term in the limit diagram (1.4), so that $\Gamma_{i}$ and hence $\Sigma_{i}$ both map to $w^{i-j}$ in $K_{0}(K)$.

Let $\Phi_{j}$ denote the Thom isomorphism $\widetilde{K}_{0}(M U(j)) \rightarrow K_{0}(B U(j))$.

Proposition 6.3. Under the composition

$$
K_{0}(B U(j)) \stackrel{\Phi_{j}^{-1}}{\longrightarrow} \widetilde{K}_{0}(M U(j)) \stackrel{\iota_{*}}{\longrightarrow} \widetilde{K}_{0}(B U),
$$

$\Sigma_{k}$ maps to $(-1)^{k}\left(B_{k} / k\right)\left(\Sigma_{k+j}-\Sigma_{j}\right)$, if $j>k$.

Proof. By Corollary 6.2, $t_{*} \Phi_{j}^{-1}\left(\Sigma_{k}\right)$ maps to $(-1)^{k}\left(B_{k} / k\right)\left(w^{k}-1\right) \in K_{0}(K)$.

Since $j-1 \geq k$, the element $\Sigma_{k}$ belongs to $K_{0}(B U(j-1))$ so that the diagram

$$
\begin{gathered}
K_{0}(B U(j-1)) \stackrel{\Phi_{J-1}^{-1}}{\longrightarrow} \widetilde{K}_{0}\left(S^{2} \wedge M U(j-1)\right) \\
\downarrow \\
K_{0}(B U(j)) \stackrel{\Phi_{J}^{-1}}{\longrightarrow} \widetilde{K}_{0}(M U(j)) \stackrel{\iota_{*}}{\longrightarrow} \widetilde{K}_{0}(B U)
\end{gathered}
$$

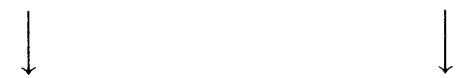

shows that $t_{*} \Phi_{j}^{-1}\left(\Sigma_{k}\right)$ is the image of $\Phi_{j-1}^{-1}\left(\Sigma_{k}\right) \in \widetilde{K}_{0}\left(S^{2} \wedge M U(j-1)\right)$ and hence is diagonal primitive. Thus $t_{*} \Phi_{j}^{-1}\left(\Sigma_{k}\right)$ is a rational linear combination of the $\Sigma_{i}$. It follows that

$$
t_{*} \Phi_{j}^{-1}\left(\Sigma_{k}\right)=(-1)^{k}\left(B_{k} / k\right)\left(\Sigma_{k+j}-\Sigma_{j}\right) .
$$


This result is a version of a standard $e$-invariant calculation; see Proposition 19.30 of [52] for example. We now generalize it to show how the elements of Definition 5.4 can be related to the Thom isomorphism.

Let $K=\left(k_{r}, \ldots, k_{1}\right)$ be an admissible sequence of natural numbers, in the sense of Definition 4.8, and let $k>|K|$. The element $\Delta_{k, K} \in K_{0}(B U)$, being a linear combination of the $\Sigma_{i}$ with $i \leq k+|K|$, belongs to $K_{0}(B U(j))$ if $j \geq k+|K|$.

Proposition 6.4. If $j>k+|K|$ then $t_{*} \Phi_{j}^{-1}\left(\Delta_{k, K}\right)=\Delta_{j,(k) K}$, where $(k) K$ is the sequence $\left(k, k_{r}, \ldots, k_{1}\right)$, as in Definition 3.2.

Proof. We use induction on $r=\operatorname{len}(K)$. The case $r=0$ is Proposition 6.3.

For the induction step note that by Proposition 5.5

$$
\Delta_{k, K(i)}=\lambda_{k}\left(\Delta_{k, K+i}-\Delta_{k, K}\right),
$$

where $\lambda_{k}=(-1)^{k} B_{k} / k$, so that

$$
t_{*} \Phi_{j}^{-1}\left(\Delta_{k, K(i)}\right)=\lambda_{k}\left(t_{*} \Phi_{j}^{-1}\left(\Delta_{k, K+i}\right)-t_{*} \Phi_{j}^{-1}\left(\Delta_{k, K}\right)\right),
$$

which we may inductively assume equals $\lambda_{k}\left(\Delta_{j,(k) K+i}-\Delta_{j,(k) K}\right)$. But, by Proposition 5.5 again, this is $\Delta_{j,(k) K(i)}$. The inequalities of the induction statement are sufficient to guarantee the validity of this argument. We interpret $\Delta_{j,()+i}$ as $\Delta_{j+i,()}=\Sigma_{j+i}$.

The next step toward realizing primitive elements in $K_{0}(B U)$ is to show how the $\Delta$-elements can be realized in the $K$-theory of finite $C W$-complexes. The complexes in question are constructed by iterating the Thom construction.

Definition 6.5. If $K=\left(k_{r}, \ldots, k_{1}\right)$ is an admissible sequence of positive integers, the iterated Thom complex $\left(X_{K}, f_{K}\right)$ consists of a $2|K|$-dimensional $C W$-complex $X_{K}$ and its Thom class $f_{K}: X_{K} \rightarrow B U$. We define $\left(X_{K}, f_{K}\right)$ recursively as follows.

Let $X_{(k)}=S^{2 k}$ and let $f_{(k)}$ be the Bott generator of $\pi_{2 k}(B U)$.

Suppose now that $\left(X_{K}, f_{K}\right)$ is defined and $k \geq|K|$, so that the sequence $(k) K=\left(k, k_{r}, \ldots, k_{1}\right)$ is admissible, then the iterated Thom complex corresponding to the sequence $(k) K$ is constructed as follows.

Since $\operatorname{dim} X_{K}=2|K| \leq 2 k$, the map $f_{K}$ factors uniquely as

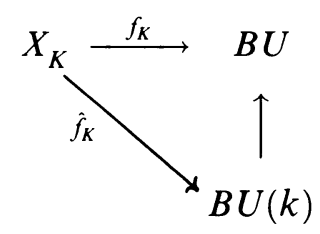

see Theorem 8 (2.6) on page 102 of [26], or 10.19 of Chapter IV of [53]. Then $X_{(k) K}$ is the Thom space of the $U(k)$-bundle over $X_{K}$ induced by $\hat{f}_{K}$ and the Thom class $f_{(k) K}$ is the composition $X_{(k) K} \rightarrow M U(k) \stackrel{t}{\rightarrow} B U$, where $t$ is the universal Thom class. 
Note that if $k>|K|$, which is necessary for $(k) K$ to be strictly admissible, then $X_{(k) K}$ is a double suspension since $\hat{f}_{K}$ factors through $B U(k-1)$.

In a particular case it may be possible to factor $f_{K}$ through $B U(j)$ for some $j$ less than $|K|$. Thus iterated Thom complexes may exist for inadmissible sequences. In this sense, $C P^{n}$ is an iterated Thom complex corresponding to the sequence $(1,1, \ldots, 1)$ of length $n$.

It follows from Lemma 12.26 of [52] that $X_{K}$ has a $C W$-complex decomposition of the form

$$
S^{2 k_{r}} \cup e^{2\left(k_{r}+k_{r-1}\right)} \cup \cdots \cup e^{2\left(k_{r}+\cdots+k_{2}\right)} \cup e^{2|K|} .
$$

Definition 6.6. The standard basis $x_{0}^{K}, x_{1}^{K}, \ldots, x_{r}^{K}$ for $K_{0}\left(X_{K}\right)$ is defined recursively by $x_{0}^{K}=1$, and $\Phi\left(x_{i+1}^{(k) K}\right)=x_{i}^{K}$ for $i=0,1, \ldots, r$, where $\Phi: \widetilde{K}_{0}\left(X_{(k) K}\right) \rightarrow K_{0}\left(X_{K}\right)$ is the Thom isomorphism.

Proposition 6.7. If the sequence $K$ is strictly admissible then

$$
\left(f_{K}\right)_{*}: K_{0}\left(X_{K}\right) \rightarrow K_{0}(B U)
$$

maps $x_{i}^{K}$ to $\Delta_{k_{r},\left(k_{r-1}, \ldots, k_{r-i+1}\right)}$ if $i \geq 1$.

Proof. The diagram

$$
\begin{array}{cc}
\widetilde{K}_{0}\left(X_{(k) K}\right) & \longrightarrow \widetilde{K}_{0}(M U(k))-\stackrel{t_{\bullet}}{\longrightarrow} \widetilde{K}_{0}(B U) \\
\Phi \downarrow \cong & \Phi_{k} \downarrow \cong \\
K_{0}\left(X_{K}\right) \stackrel{\left(f_{K}\right) \cdot}{\longrightarrow} & K_{0}(B U(k))
\end{array}
$$

in which the top row represents $\left(f_{(k) K}\right)_{*}$, shows that

$$
\left(f_{(k) K}\right)_{*}\left(x_{i+1}^{(k) K}\right)=t_{*} \Phi_{k}^{-1}\left(\left(f_{K}\right)_{*}\left(x_{i}^{K}\right)\right) .
$$

Assuming inductively that $\left(f_{K}\right)_{*}\left(x_{i}^{K}\right)=\Delta_{k_{r},\left(k_{r-1}, \ldots, k_{r-i+1}\right)}$,

$$
\begin{aligned}
\left(f_{(k) K}\right)_{*}\left(x_{i+1}^{(k) K}\right) & =t_{*} \boldsymbol{\Phi}_{k}^{-1}\left(\Delta_{k_{r},\left(k_{r-1}, \ldots, k_{r-1+1}\right)}\right) \\
& =\Delta_{k,\left(k_{r}, k_{r-1}, \ldots, k_{r-i+1}\right)},
\end{aligned}
$$

by Proposition 6.4 .

The induction is initiated by the remark that $t_{*} \Phi_{k}^{-1}(1)=\Sigma_{k}=\Delta_{k,()}$, by definition of $\Sigma_{k}$.

Definition 6.8. The standard basis $\xi_{0}^{K}, \xi_{1}^{K}, \ldots, \xi_{r}^{K}$ for $K^{0}\left(X_{K}\right)$ is defined recursively by $\xi_{0}^{K}=1$, and $\Phi\left(\xi_{i}^{K}\right)=\xi_{i+1}^{(k) K}$ for $i=0,1, \ldots, r$, where $\Phi$ : $K^{0}\left(X_{K}\right) \rightarrow \widetilde{K}^{0}\left(X_{(k) K}\right)$ is the Thom isomorphism.

Thus $f_{K}$ represents $\xi_{1}^{K}$.

The standard basis for $K^{0}\left(X_{K}\right)$ is dual to the homology basis of Definition 6.6 . 
Our final task in this section is to generalize Proposition 6.7 by providing a formula for the slant product $\xi_{i}^{K} / x_{j}^{K}=\left(\xi_{i}^{K}\right)_{*}\left(x_{j}^{K}\right)$. To do this we need the following results on the Thom isomorphism.

Lemma 6.9. Let $M$ be the Thom complex of a bundle over $B$ which is oriented with respect to $K$-theory. Suppose that $K_{0}(B)$ is torsion-free, so that $K_{0}(B)$ is a coalgebra with coproduct $\Delta: K_{0}(B) \rightarrow K_{0}(B) \otimes K_{0}(B)$.

Let $\delta: M \rightarrow M \wedge B_{+}$be the reduced diagonal [33], and let $\Phi: \widetilde{K}_{0}(M) \rightarrow$ $K_{0}(B)$ denote the Thom isomorphism.

Then we have

$$
\delta_{*}(x)=\left(\Phi^{-1} \otimes 1\right) \Delta \Phi(x)
$$

in $\widetilde{K}_{0}\left(M \wedge B_{+}\right) \cong \widetilde{K}_{0}(M) \otimes K_{0}(B)$.

In particular, if $\Phi(x) \in K_{0}(B)$ is primitive then

$$
\delta_{*}(x)=x \otimes 1+\Phi^{-1}(1) \otimes \Phi(x) .
$$

Proof. The following diagram commutes:

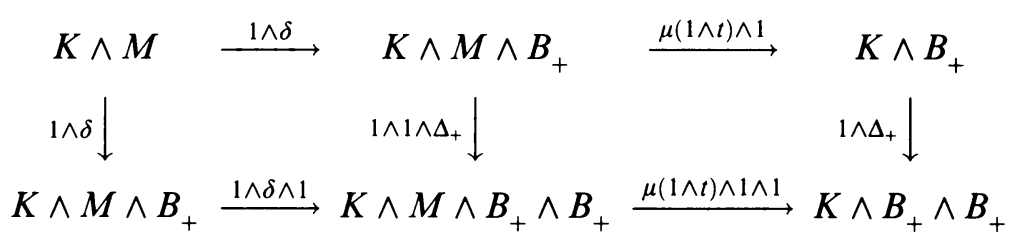

where $\Delta_{+}: B_{+} \rightarrow(B \times B)_{+}=B_{+} \wedge B_{+}$is the diagonal, which induces the coproduct on $K_{0}(B)$, and $t \in \widetilde{K}^{0}(M)$ is the Thom class.

The induced diagram of homotopy groups is

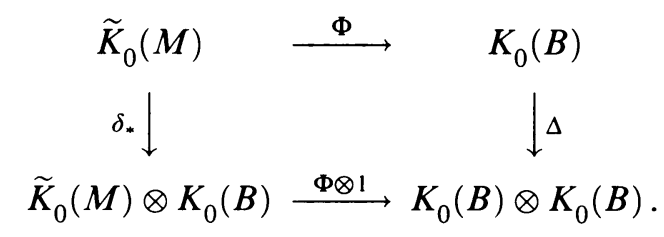

The result follows.

Proposition 6.10. Given the assumptions of the lemma, let $\xi \in K^{0}(B)$ be represented as a map $\xi: B_{+} \rightarrow K$ and $\Phi(\xi) \in \widetilde{K}^{0}(M)$ as a map $\Phi(\xi): M \rightarrow K$. If $x \in \widetilde{K}_{0}(M)$ is such that $\Phi(x) \in K_{0}(B)$ is diagonal primitive, then

$$
\Phi(\xi)_{*}(x)=t_{*}(x) \cdot \xi_{*}(1)+t_{*}\left(\Phi^{-1}(1)\right) \cdot \xi_{*}(\Phi(x))
$$

in $K_{0}(K)$.

In particular, if $\xi \in \widetilde{K}^{0}(B)$ then

$$
\Phi(\xi)_{*}(x)=t_{*}\left(\Phi^{-1}(1)\right) . \xi_{*}(\Phi(x)) .
$$

Proof. The map $\Phi(\xi): M \rightarrow K$ is the composition

$$
M \stackrel{\delta}{\longrightarrow} M \wedge B_{+} \stackrel{t \wedge \xi}{\longrightarrow} K \wedge K \stackrel{\mu}{\longrightarrow} K .
$$


Proposition 6.11. If the sequence $K=\left(k_{r}, \ldots, k_{1}\right)$ is strictly admissible, and $x_{j}^{K} \in K_{0}\left(X_{K}\right), \xi_{i}^{K} \in K^{0}\left(X_{K}\right)$ are elements of the standard bases then $\left(\xi_{i}^{K}\right)_{*}$ : $K_{0}\left(X_{K}\right) \rightarrow K_{0}(K)$ maps $x_{j}^{K}$ to

$$
\begin{cases}w^{k_{r}+\cdots+k_{r-i+1}} W_{\left(k_{r-i}, \ldots, k_{r-j+1}\right)}(w), & \text { if } 1 \leq i \leq j, \\ 0, & \text { if } i>j \geq 0 .\end{cases}
$$

Proof. Since the sequence $K$ is strictly admissible, all the Thom complexes $X_{\left(k_{r-s}, \ldots, k_{1}\right)}$ are suspensions, so that every element of their reduced $K$-homology groups is primitive. We may therefore repeatedly apply the previous proposition to obtain

$$
\begin{aligned}
\left(\xi_{i}^{K}\right)_{*}\left(x_{j}^{K}\right) & =w^{k_{r}}\left(\xi_{i-1}^{\left(k_{r-1}, \ldots, k_{1}\right)}\right)_{*}\left(x_{j-1}^{\left(k_{r-1}, \ldots, k_{1}\right)}\right) \\
& =w^{k_{r}+k_{r-1}}\left(\xi_{i-2}^{\left(k_{r-2}, \ldots, k_{1}\right)}\right)_{*}\left(x_{j-2}^{\left(k_{r-2}, \ldots, k_{1}\right)}\right) \\
& =\ldots,
\end{aligned}
$$

where we note that if $t \in K^{0}(M)$ is the Thom class of a $U(k)$-bundle then $t_{*}\left(\Phi^{-1}(1)\right)=w^{k} \in K_{0}(K)$.

This reduction will stop for one of two reasons.

First, we may reach $x_{1}^{\left(k_{r-j+1}, \ldots, k_{1}\right)}$, whose image under $\Phi$ is 1 , which is not primitive. This occurs if $j<i$, in which case

$$
\left(\xi_{i-j+1}^{\left(k_{r-j+1}, \ldots, k_{1}\right)}\right)_{*}\left(x_{1}^{\left(k_{r-j+1}, \ldots, k_{1}\right)}\right)=0,
$$

so that $\left(\xi_{i}^{K}\right)_{*}\left(x_{j}^{K}\right)=0$.

If on the other hand $j \geq i$, we have

$$
\left(\xi_{i}^{K}\right)_{*}\left(x_{j}^{K}\right)=w^{k_{r}+\cdots+k_{r-i+2}}\left(\xi_{1}^{\left(k_{r-i+1}, \ldots, k_{1}\right)}\right)_{*}\left(x_{j-i+1}^{\left(k_{r-i+1}, \ldots, k_{1}\right)}\right) .
$$

But, as a map into the spectrum $K, \xi_{1}^{\left(k_{r-i+1}, \ldots, k_{1}\right)}$ is the composition of

$$
f_{\left(k_{r-i+1}, \ldots, k_{1}\right)}: X_{\left(k_{r-i+1}, \ldots, k_{1}\right)} \rightarrow B U
$$

with the limit map $B U \rightarrow K$.

Now by Proposition 6.7

$$
\left(f_{\left(k_{r-i+1}, \ldots, k_{1}\right)}\right)_{*}\left(x_{j-i+1}^{\left(k_{r-i+1}, \ldots, k_{1}\right)}\right)=\Delta_{k_{r-i+1},\left(k_{r-i}, \ldots, k_{r-j+1}\right)}
$$

in $K_{0}(B U)$, and

$$
l\left(\Delta_{k_{r-i+1},\left(k_{r-i}, \ldots, k_{r-j+1}\right)}\right)=w^{k_{r-i+1}} W_{\left(k_{r-i}, \ldots, k_{r-j+1}\right)}(w) .
$$

The result follows.

Corollary 6.12. Under the conditions of the proposition, the induced map $\left(\xi_{i}^{K}\right)_{*}$ : $K_{0}\left(X_{K}\right) \rightarrow K_{0}(B U)$ maps $x_{j}^{K}$ to

$$
\begin{cases}\Delta_{k_{r}+\cdots+k_{r-i+1},\left(k_{r-i}, \ldots, k_{r-j+1}\right)}, & \text { if } 1 \leq i \leq j, \\ 0, & \text { if } i>j \geq 0 .\end{cases}
$$


Proof. Since $K$ is strictly admissible, $X_{K}$ is a suspension and thus the element $\left(\xi_{i}^{K}\right)_{*}\left(x_{j}^{K}\right) \in K_{0}(B U)$ is diagonal primitive. The result now follows from the definition of the $\Delta$-elements.

\section{HyPERSURFACES AND DUALITY}

This section is devoted to a geometric construction of elements in the stable homotopy of $B U$. It is this technique which, in $\S 8$, will enable us to realize the elements of $P_{*} K_{0}(B U)$ which we constructed in $\S 5$. We will use it again in $\S \S 9$ and 10.

After describing the construction we will show how $S$-duality may be used to provide a formula for the $K$-theory Hurewicz images of the stable homotopy classes generated by our method.

Suppose that $A$ is a subcomplex of $S^{2 n+1}$. Let $N$ denote a regular neighborhood of $A$, so that $N$ retracts onto $A$. We may give $N$ a smooth structure so that the boundary of $N$ is a smooth hypersurface $M \subset S^{2 n+1}$ [22]. We retract $N$ onto a smaller neighborhood $N^{\prime}$ so that $N=N^{\prime} \cup[0,1] \times M$. If we identify $A$ with $N^{\prime}$ and let $B$ denote the complement of $N$ in $S^{2 n+1}$, we have a decomposition of the sphere as

$$
S^{2 n+1}=A \cup[0,1] \times M \cup B,
$$

in which $[0,1] \times M$ intersects $A$ in $\{0\} \times M$ and $B$ in $\{1\} \times M$.

Let $i: M \rightarrow A$ and $j: M \rightarrow B$ denote the boundary inclusions. Given two maps $f: A \rightarrow B U$ and $g: B \rightarrow B U$, or equivalently elements of $\widetilde{K}^{0}(A)$ and $\widetilde{K}^{0}(B)$, we may combine them as the composition

$$
f \oplus g: M \stackrel{\Delta}{\longrightarrow} M \times M \stackrel{i \times j}{\longrightarrow} A \times B \stackrel{f \times g}{\longrightarrow} B U \times B U \stackrel{\omega}{\longrightarrow} B U,
$$

where $\omega: B U \times B U \rightarrow B U$ is the Whitney sum map.

As a hypersurface, $M$ is a framed manifold and the framed bordism class of $f \oplus g: M \rightarrow B U$ defines an element of $\pi_{2 n}^{S}(B U)$.

Let $c: S^{2 n+1} \rightarrow S^{1} \wedge M$ denote the Pontrjagin-Thom collapse (see the proof of Theorem 12.30 of [52]) so that the stable homotopy class represented by $[M, f \oplus g]$ is that of the composition

$$
S^{2 n+1} \stackrel{c}{\longrightarrow} S^{1} \wedge M \stackrel{1 \wedge(f \oplus g)}{\longrightarrow} S^{1} \wedge B U .
$$

Lemma 7.1. With the preceding notation the composition

$$
\rho_{A}: S^{2 n+1} \stackrel{c}{\longrightarrow} S^{1} \wedge M \stackrel{1 \wedge i \wedge j}{\longrightarrow} S^{1} \wedge A \wedge B
$$

is an $S$-duality map, in the sense of page 326 of [52].

Proof. Though this result is well known (see for example [45]), the only complete proof in the literature seems to be that in [18].

Lemma 7.1 provides us with an isomorphism

$$
D: \widetilde{K}^{0}(A) \rightarrow \widetilde{K}_{2 n}(B) \cong \widetilde{K}_{0}(B),
$$


which sends an element $\xi$ of $\widetilde{K}^{0}(A)$, considered as a map $\xi: A \rightarrow K$, to the element of $\widetilde{K}_{2 n}(B)$ represented by the composition

$$
S^{2 n+1} \stackrel{\rho}{\longrightarrow} S^{1} \wedge A \wedge B \stackrel{1 \wedge \xi \wedge 1}{\longrightarrow} S^{1} \wedge K \wedge B .
$$

Similarly, we have an isomorphism

$$
D: \widetilde{K}^{0}(B) \rightarrow \widetilde{K}_{2 n}(A) \cong \widetilde{K}_{0}(A) .
$$

Lemma 7.2. Suppose that $\rho: S^{2 n} \rightarrow A \wedge B$ is an $S$-duality map and that $\widetilde{K}_{0}(A)$ is torsion-free with basis $x_{1}, x_{2}, \ldots, x_{r}$. Let the Kronecker dual basis for $\widetilde{K}_{0}(A)$ be denoted by $\xi_{1}, \xi_{2}, \ldots, \xi_{r}$, so that $D \xi_{1}, D \xi_{2}, \ldots, D \xi_{r}$ is a basis for $\widetilde{K}_{2 n}(B) \cong$ $\widetilde{K}_{0}(B)$. The induced homomorphism $\rho_{*}: \widetilde{K}_{0}\left(S^{2 n}\right) \rightarrow \widetilde{K}_{0}(A \wedge B) \cong \widetilde{K}_{0}(A) \otimes \widetilde{K}_{0}(B)$ sends the generator $g_{n}$ to $\sum_{i=1}^{r} x_{i} \otimes D \xi_{i}$.

Proof. The work of Dold and Puppe [18] provides a more general setting for this result. In their terminology $\widetilde{K}_{0}$ is a monoidal functor and $A$ is $\widetilde{K}_{0}$-flat. It follows from Corollary 2.4 of [18] that $\rho_{*}$ is the coevaluation for the duality between $\widetilde{K}_{0}(A)$ and $\widetilde{K}_{0}(B)$. The lemma follows from Example 1.4 of [18].

We can now prove the result which will enable us to compute the $K$-theory Hurewicz image of stable homotopy classes realized on hypersurfaces.

Proposition 7.3. Let $A$ and $B$ be complementary subcomplexes of $S^{2 n+1}$, such that $K_{0}(A)$ is torsion-free, with $M$ the hypersurface which separates them. Let $c: S^{2 n+1} \rightarrow S^{1} \wedge M$ be the Pontrjagin-Thom collapse.

The composition

$\widetilde{K}_{0}\left(S^{2 n}\right) \cong \widetilde{K}_{1}\left(S^{2 n+1}\right) \stackrel{c_{*}}{\longrightarrow} \widetilde{K}_{1}\left(S^{1} \wedge M\right) \stackrel{(1 \wedge(i \times j))}{\longrightarrow} \widetilde{K}_{1}\left(S^{1} \wedge(A \times B)\right) \cong \widetilde{K}_{0}(A \times B)$ maps the generator to $\sum_{i=1}^{r} x_{i} \otimes D \xi_{i}$, where $x_{1}, x_{2}, \ldots, x_{r}$ is a basis for $\widetilde{K}_{0}(A)$ and $\xi_{1}, \xi_{2}, \ldots, \xi_{r}$ is the dual basis for $\widetilde{K}^{0}(A)$.

Proof. If $C X=[0,1] \wedge X$ denotes the cone on $X$, let

$$
A * B=C A \times B \cup A \times C B \subset C A \times C B
$$

denote the join of $A$ and $B$; see page 482 of [53]. The Hopf construction, when applied to the identity map, gives us a map $\Gamma 1: A * B \rightarrow S^{1} \wedge(A \times B)$, which, composed with the suspension of the quotient map $A \times B \rightarrow A \wedge B$, determines a homotopy equivalence between $A * B$ and $S^{1} \wedge A \wedge B$; see Lemma 8.15 of Chapter X of [53].

As we saw above, we can decompose $S^{2 n+1}$ into the union of $A,[0,1] \times \mathrm{M}$, and $B$.

We define $\gamma: S^{2 n+1} \rightarrow A * B$ as follows. If $a \in A$ let $\gamma(a)=a \times *$ in $A \times C B$; similarly, $\gamma(b)=* \times b$ in $C A \times B$, while on $[0,1] \times M$,

$$
\gamma(t \times m)= \begin{cases}i(m) \times(2 t \wedge j(m)) \text { in } A \times C B, & \text { if } t \leq \frac{1}{2}, \\ (2-2 t \wedge i(m)) \times j(m) \text { in } C A \times B, & \text { if } t \geq \frac{1}{2} .\end{cases}
$$


Consider the following diagram:

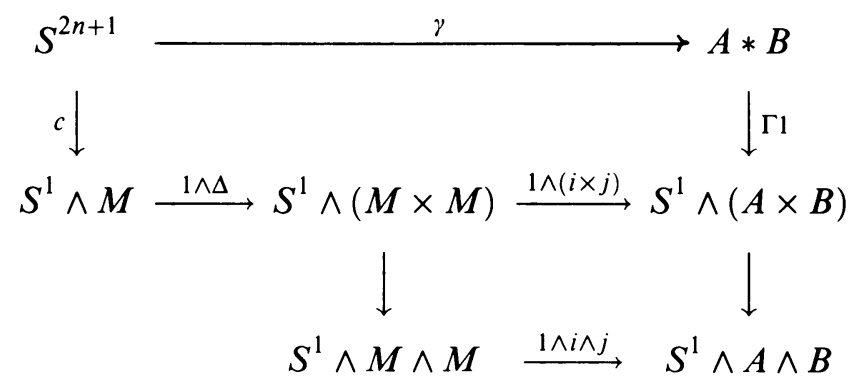

which is easily seen to commute.

The result now follows from Lemmas 7.1 and 7.2 since the map

$$
\Gamma 1_{*}: \widetilde{K}_{1}(A * B) \rightarrow \widetilde{K}_{1}\left(S^{1} \wedge(A \times B)\right) \cong \widetilde{K}_{0}(A \wedge B) \oplus \widetilde{K}_{0}(A) \oplus \widetilde{K}_{0}(B)
$$

maps onto the summand $\widetilde{K}_{0}(A \wedge B)$.

Corollary 7.5. Suppose that, with the notation of the proposition, $f$ and $g$ are maps from $A$ and $B$, respectively, to $B U$. The $K$-theory Hurewicz image of the class $[M, f \oplus g]$ is $\sum_{i=1}^{r} f_{*}\left(x_{i}\right) g_{*}\left(D \xi_{i}\right)$.

We may apply other homology theories to the diagram (7.4) to obtain the corresponding Hurewicz images. In particular, if we use the theory $\pi_{*}^{S}()$, the generator maps to $[M, f \oplus g]$ itself. This observation will be useful in $\S 8$.

In order to make use of the formula of the corollary we need to be able to evaluate $g_{*}\left(D \xi_{i}\right)$. The next proposition shows how to do this.

Proposition 7.6. Let $\rho: S^{2 n} \rightarrow A \wedge B$ be an $S$-duality map with $\xi \in \widetilde{K}^{0}(A)$ and $y \in \widetilde{K}^{0}(B)$, so that $D \xi \in \widetilde{K}_{0}(B)$ and $D y \in \widetilde{K}_{0}(A)$. Then

$$
y_{*}(D \xi)=w^{n} \chi\left(\xi_{*}(D y)\right) \in K_{0}(K)
$$

where $\xi_{*}$ and $y_{*}$ are the homomorphisms induced in $K$-theory by the maps $\xi: A \rightarrow K$ and $y: B \rightarrow K$, and $\chi: K_{0}(K) \rightarrow K_{0}(K)$ is the ring homomorphism $w \mapsto w^{-1}$ induced by swapping the two copies of the spectrum.

Proof. A simple diagram chase.

\section{REALIZING THE KUMMER CONGRUENCES IN STABLE HOMOTOPY}

In this section we show that the elements $\Xi_{k, j, K} \in P_{*} K_{0}(B U)$ which were constructed in $\S 5$ using the Kummer congruences can be realized in the stable homotopy of $B U$.

In $\S 5$ we expressed the primitives $\Xi_{k, j, K}$ in terms of the $\Delta$-elements and the $\nabla$-elements. In $\S 6$ we showed that the $\Delta$-elements could be realized in the $K$-theory of iterated Thom complexes. The only remaining component of the proof of the main theorem of this section is to show how the $\nabla$-elements may be realized in the $K$-theory of the $S$-duals of iterated Thom complexes. 
Suppose that the iterated Thom complex $X_{K}$, corresponding to the strictly admissible sequence $K=\left(k_{r}, \ldots, k_{1}\right)$, embeds in $S^{2 n}$, and that $Y_{K}$ is the complement in $S^{2 n+1}$. Thus $Y_{K}$ has the homotopy type of a suspension and the map $\rho: S^{2 n+1} \rightarrow S^{1} \wedge X_{K} \wedge Y_{K}$ of Lemma 7.1 is an $S$-duality map.

Proposition 8.1. Let $y_{1}, y_{2}, \ldots, y_{r} \in \widetilde{K}^{0}\left(Y_{K}\right)$ be the basis defined by $D y_{j}=x_{j}$, where $x_{j}$ is the standard basis element of Definition 6.6. Then $\left(y_{j}\right)_{*}: K_{0}\left(Y_{K}\right) \rightarrow$ $K_{0}(B U)$ maps $D \xi_{i}$ to

$$
\begin{cases}\nabla_{n-|K|+k_{r-j}+\cdots+k_{1},\left(k_{r-i}, \ldots, k_{r-j+1}\right)}, & \text { if } 1 \leq i \leq j \\ 0, & \text { if } i>j>0 .\end{cases}
$$

Proof. By Propositions 6.11 and 7.6, $\left(y_{j}\right)_{*}: K_{0}\left(Y_{K}\right) \rightarrow K_{0}(K)$ maps $D \xi_{i}$ to

$$
\begin{cases}w^{n-k_{r}-\cdots-k_{r-i+1}} W_{\left(k_{r-i}, \ldots, k_{r-j+1}\right)}\left(w^{-1}\right), & \text { if } 1 \leq i \leq j, \\ 0, & \text { if } i>j>0 .\end{cases}
$$

But, since $Y_{K}$ is a suspension, $\left(y_{j}\right)_{*}\left(D \xi_{i}\right) \in K_{0}(B U)$ is diagonal primitive and thus a rational linear combination of the $\Sigma_{k}$. The result now follows from the definition of the $\nabla$-elements, 5.4.

Theorem 8.2. Let $K$ be a strictly admissible sequence, as in Definition 4.8, and let $n=k+j+|K|$, where $k, j>|K|$. The element $\Xi_{k, j, K} \in P_{n} K_{0}(B U)$ of Definition 5.7 is in the image of the stable Hurewicz homomorphism.

Proof. Let $X_{(k) K}$ be the iterated Thom complex corresponding to the strictly admissible sequence $(k) K$; see Definition 6.5. The complex $X_{(k) K}$ has dimension $2 k+2|K|$ and is $(2 k-1)$-connected. By a result of Stallings [50] (see also Theorem 12.1 of [24]) it may be embedded up to homotopy type in $S^{4|K|+2 k+1}$, and hence in $S^{2 n+1}$.

Let $Y$ be the complement of $X_{(k) K}$ in $S^{2 n+1}$. Since $j>|K|$ it follows that $Y$ has the homotopy type of a suspension.

Let $f=f_{(k) K}: X_{(k) K} \rightarrow B U$ be the Thom class and let $g: Y \rightarrow B U$ be chosen so that the dual $K$-theory class $D g$ is the element $x_{r+1}^{(k) K}$ of the standard basis for $K_{0}\left(X_{(k) K}\right)$.

We will show that, if $M$ is the hypersurface separating $X_{(k) K}$ and $Y$, the element $\Psi_{k, j, K}$ of $\pi_{2 n}^{S}(B U)$ represented by $f \oplus g: M \rightarrow B U$ maps to $\Xi_{k, j, K} \in$ $P_{n} K_{0}(B U)$ under the Hurewicz homomorphism.

By Corollary 7.5 the image of $\Psi_{j, k, K}$ is $\sum_{i=1}^{r+1} f_{*}\left(x_{i}\right) g_{*}\left(D \xi_{i}\right)$.

But by Corollary $6.12 f_{*}\left(x_{i}\right)=\Delta_{k,\left(k_{r}, \ldots, k_{r-i+2}\right)}$, and by Proposition $8.1 g_{*}\left(D \xi_{i}\right)$ $=\nabla_{j,\left(k_{r-i+1}, \ldots, k_{1}\right)}$.

Thus

$$
\sum_{i=1}^{r+1} f_{*}\left(x_{i}\right) g_{*}\left(D \xi_{i}\right)=\sum_{I J=K} \Delta_{k, I} \nabla_{j, J},
$$

which, by Theorem 5.9, equals $\Xi_{k, j, K}$. 
It is natural to ask whether we can realize any more primitives in $K_{0}(B U)$ by varying the maps $f$ and $g$ in the proof of Theorem 8.2. Since our formula for the Hurewicz image of $[M, f \oplus g]$ is bilinear in $f$ and $g$, it is sufficient to consider the case where $f$ represents $\xi_{s} \in K^{0}\left(X_{(k) K}\right)$ and, in the notation of Proposition 8.1, $g$ represents $y_{t} \in \widetilde{K}^{0}(Y)$. (In the proof of Theorem 8.2 we had $s=1$ and $t=r+1$.) Then

$$
f_{*}\left(x_{i}\right)=\Delta_{k+k_{r}+\cdots+k_{r-s+2},\left(k_{r-s+1}, \ldots, k_{r-i+2}\right)}, \quad i \geq s,
$$

and

$$
g_{*}\left(D \xi_{i}\right)=\nabla_{j+k_{r-t+1}+\cdots+k_{1},\left(k_{r-i+1}, \ldots, k_{r-t+2}\right)}, \quad i \leq t .
$$

Thus, if $s>t$, the Hurewicz image of $[M, f \oplus g]$ is zero; otherwise it is

$$
\Xi_{k+k_{r}+\cdots+k_{r-s+2}, j+k_{r-t+1}+\cdots+k_{1},\left(k_{r-s+1}, \ldots, k_{r-t+2}\right)},
$$

which does not provide any improvement on Theorem 8.2.

It is clear that $m_{K} \Xi_{k, j, K}$ is an integral bilinear combination of the $\Sigma_{i}$ in $P_{*} K_{0}(B U)$ for some minimal positive integer $m_{K}$. We would like to deduce the same divisibility result for the corresponding combination of the $\Sigma_{i}$ in $\pi_{*}^{S}(B U)$. Unfortunately, Theorem 8.2 tells us only that this is so modulo torsion.

We complete this section by extending our methods to show, in Theorem 8.8 , that the expected divisibility does indeed occur in $\pi_{*}^{S}(B U)$, although for simplicity we replace $m_{K}$ by an integer $t_{K}$ which is, in general, larger.

We need first some preparatory constructions involving the iterated Thom complexes $X_{K}$. For a strictly admissible sequence $K$ the complex $X_{K}$ is a suspension so that for any integer $t$ and map $f: X_{K} \rightarrow Z$ we can define the $t$-fold sum of $f$ with itself, written $t f$, by using the cogroup structure. In particular, $t: X_{K} \rightarrow X_{K}$ will denote $t .1$ so that $t f=t^{*}(f)$. We let $S^{-i} X$ stand for an $i$-fold desuspension of $X$.

Let $o(k)=\frac{1}{2} m(k)$ denote the denominator of $B_{k} / k$ and hence also the $J$-order of the Bott generator of $\pi_{2 k}(B U)$ [2].

Definition 8.3. If $K=\left(k_{r}, \ldots, k_{1}\right)$ is a sequence of positive integers, the iterated wedge $V_{K}$ is defined as

$$
V_{K}=S^{2 k_{r}} \vee S^{2\left(k_{r}+k_{r-1}\right)} \vee \cdots \vee S^{2\left(k_{r}+\cdots+k_{2}\right)} \vee S^{2|K|} .
$$

Let the elements $v_{1}^{K}, v_{2}^{K}, \ldots, v_{r}^{K}$ in $\pi_{*}^{S}\left(V_{K}\right)$ be such that $v_{i}^{K}$ is represented by the inclusion $S^{2\left(k_{r}+\cdots+k_{r-i+1}\right)} \subset V_{K}$. Let the elements $\nu_{1}^{K}, \nu_{2}^{K}, \ldots, \nu_{r}^{K} \in$ $\pi_{S}^{*}\left(V_{K}\right)$ be such that $\nu_{i}^{K}$ is represented by the projection $V_{K} \rightarrow S^{2\left(k_{r}+\cdots+k_{r-i+1}\right)}$.

Note that $\pi_{*}^{S}\left(V_{K}\right)$ is free over $\pi_{*}^{S}$, and the $v_{i}^{K}$ and $\nu_{i}^{K}$ are dual bases.

Definition 8.4. If $K=\left(k_{r}, \ldots, k_{1}\right)$ is a sequence of positive integers we let $o_{K}$ denote the least common multiple of $o\left(k_{r}\right), o\left(k_{r}+k_{r-1}\right), \ldots, o\left(k_{r}+\cdots+k_{2}\right)$, and $o(|K|)$, and define the integer $t_{K}$ inductively by

$$
t_{()}=1 \text { and } t_{(k) K}=o_{(k) K} t_{K} \text {. }
$$


Any element of $K^{0}\left(V_{K}\right)$ has $J$-order dividing $o_{K}$.

It follows from Definition 3.3 that $t_{K} W_{K}(w)$ is an integer polynomial for every sequence $K$. In general $t_{K}$ is an overestimate for the denominator of $W_{K}(w)$, which is also the denominator of $\Xi_{k, j, K}$. However, $t_{K}$ is equal to the denominator for all sequences of length one and for those sequences of length two for which no cancellation takes place among the Bernoulli numbers involved. For sequences of greater length our results could be improved by making more precise estimates of the $J$-orders involved in the proofs.

Lemma 8.5. Let $K$ be strictly admissible. There are maps $a_{K}: V_{K} \rightarrow X_{K}$ and $b_{K}: X_{K} \rightarrow V_{K}$, both of which are double suspensions, such that the composite $a_{K} b_{K}: X_{K} \rightarrow X_{K}$ is homotopic to $t_{\left(k_{r-1}, \ldots, k_{1}\right)}$.

Proof. We proceed by induction on len $(K)$.

For a sequence $(k)$ of length one we let both $a_{(k)}$ and $b_{(k)}$ be the identity on $S^{2 k}$.

Now suppose that we have constructed $a_{K}$ and $b_{K}$, and that $k>|K|$. The complex $X_{(k) K}$ is the Thom complex of $\hat{f}_{K}: X_{K} \rightarrow B U(k)$, which factors through $B U(k-1)$. Thus, by Lemma 5.15 of [34], $S^{-2} X_{(k) K}$ is the cofiber of the map $J\left(\hat{f}_{K}\right): S^{2 k-3} \wedge X_{K} \rightarrow S^{2 k-2}$, obtained by adjointing the composition

$$
S^{-1} X_{K} \stackrel{\hat{f}_{K}}{\longrightarrow} U(k-1) \stackrel{J}{\longrightarrow} \Omega^{2 k-2} S^{2 k-2} .
$$

Furthermore, since $a_{K}$ desuspends and $\left(a_{K}\right)^{*} \hat{f}_{K}$ over $V_{K}$ has $J$-order dividing $t_{K}$, there is a cofiber diagram

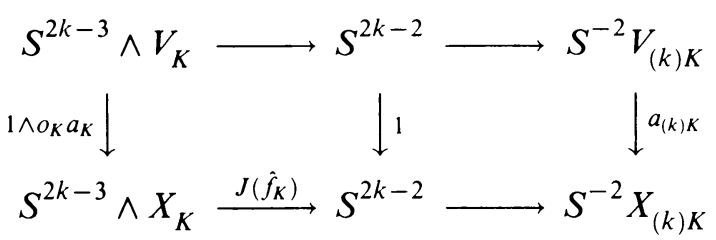

which defines $a_{(k) K}$.

Similarly $\left(t_{\left(k_{r-1}, \ldots, k_{1}\right)}\right)^{*} \hat{f}_{K}=\left(b_{K}\right)^{*}\left(a_{K}\right)^{*} \hat{f}_{K}$ by our induction hypothesis so that $\hat{f}_{K}$ has $J$-order dividing $o_{K} t_{\left(k_{r-1}, \ldots, k_{1}\right)}=t_{K}$. But $X_{K}$ is a double suspension, so the codegree of $\hat{f}_{K}$ also divides $t_{K}$; see [29] or [17]. Thus there is a cofiber diagram

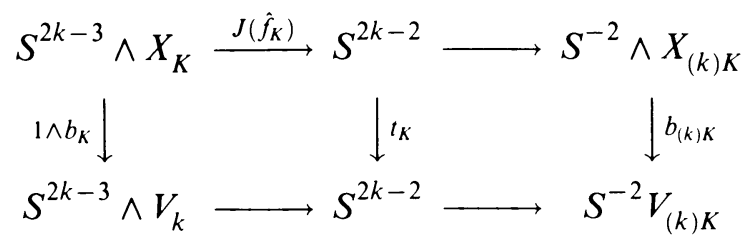

which defines $b_{(k) K}$. 
We can now amalgamate the two cofiber diagrams, suspend once, and deform all cogroup structures onto the new suspension coordinates. This yields the diagram

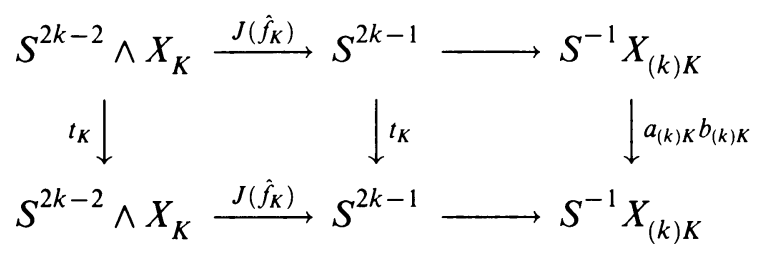

which completes the induction.

Our next step is to mimic the proof of Theorem 8.2 by embedding $V_{(k) K}$ in $S^{2 n+1}$, where $n=k+j+|K|$ with $j>|K|$. The complement $W$ admits a homotopy equivalence

$$
W \simeq S^{2 j} \vee S^{2\left(j+k_{1}\right)} \vee \cdots \vee S^{2\left(j+k_{1}+\cdots+k_{r-1}\right)} \vee S^{2(j+|K|)} .
$$

Thus, for dimensional reasons, both $W$ and the complement $Y$ of $X_{(k) K}$ are stable complexes, and we can realize the dual of $b_{(k) K}$ as a map $d: W \rightarrow Y$.

Lemma 8.6. With the notation of $\S 7$, the composition

$$
\pi_{2 n}^{S}\left(S^{2 n}\right) \stackrel{\left(\rho_{V}\right)_{*}}{\longrightarrow} \pi_{2 n}^{S}\left(V_{(k) K} \wedge W\right) \stackrel{\left(a_{(k) K} \wedge d\right)_{*}}{\longrightarrow} \pi_{2 n}^{S}\left(X_{(k) K} \wedge Y\right)
$$

maps the generator to $t_{K}$ times the image of the generator under

$$
\rho_{X}: \pi_{2 n}^{S}\left(S^{2 n}\right) \rightarrow \pi_{2 n}^{S}\left(X_{(k) K} \wedge Y\right) .
$$

Proof. Using Lemma 8.5 it suffices to note that the diagram

$$
\begin{gathered}
S^{2 n+1} \stackrel{\rho_{X}}{\longrightarrow} S^{1} \wedge X_{(k) K} \wedge Y \\
\rho_{V} \wedge V_{(k) K} \wedge W \stackrel{1 \wedge d}{\longrightarrow} S^{1} \wedge V_{(k) K} \wedge Y \text { 位 }
\end{gathered}
$$

is stably homotopy commutative. This follows from standard properties of duality; see [52].

Corollary 8.7. The element of $\pi_{2 n}^{S}(B U)$ constructed as in $\S 7$ from the hypersurface $H$ separating $V_{(k) K}$ and $W$, using the maps $F=\left(a_{(k) K}\right)^{*} f: V_{(k) K} \rightarrow B U$ and $G=d^{*} g: W \rightarrow B U$, is precisely $t_{K} \Psi_{k, j, K}$. Thus it has Hurewicz image $t_{K} \Xi_{k, j, K}$ in $P_{n} K_{0}(B U)$.

Note that the hypersurface $H$ is a connected sum of a product of spheres.

Theorem 8.8. There are integers $q_{i}$, for $i=1, \ldots, r$, such that

$$
t_{K} \Psi_{k, j, K}=\sum_{i=1}^{r} q_{i} \Sigma_{k_{r}+\cdots+k_{r-i+1}} \Sigma_{n-\left(k_{r}+\cdots+k_{r-i+1}\right)}
$$


in $\pi_{2 n}^{S}(B U)$, and

$$
\Xi_{k, j, K}=\frac{1}{t_{K}} \sum_{i=1}^{r} q_{i} \Sigma_{k_{r}+\cdots+k_{r-i+1}} \Sigma_{n-\left(k_{r}+\cdots+k_{r-i+1}\right)}
$$

in $P_{n} K_{0}(B U)$.

Proof. Using the previous corollary we may represent $t_{K} \Psi_{k, j, K}$ by $F \oplus G$ : $H \rightarrow B U$. Appealing again to [18], since $\pi_{*}^{S}$ is a monoidal functor and $V_{(k) K}$ is $\pi_{*}^{S}$-flat, we see, in Lemma 7.2 , that the homomorphism

$$
\rho_{V}: \pi_{2 n}^{S}\left(S^{2 n}\right) \rightarrow \pi_{2 n}^{S}\left(V_{(k) K} \wedge W\right)
$$

maps the generator to $\sum_{i=1}^{r} v_{i} \otimes D \nu_{i}$. Thus, as in Corollary 7.5, $t_{K} \Psi_{k, j, K}=$ $\sum_{i=1}^{r} F_{*}\left(v_{i}\right) G_{*}\left(D \nu_{i}\right)$ in $\pi_{2 n}^{S}(B U)$.

By definition, $D \nu_{i}$ is represented by an inclusion $S^{2 n-\left(k_{r}+\cdots+k_{r-i+1}\right)} \subset W$, so that both $F_{*}\left(v_{i}\right)$ and $G_{*}\left(D \nu_{i}\right)$ originate unstably in $\pi_{*}(B U)$. This establishes the theorem.

\section{ANOTHER FAMILY IN THE STABLE HOMOTOPY OF $B U$}

In this section we use our machinery to produce another family of primitives in the $K$-theory of $B U$ and to realize this family in stable homotopy. Both the number theory and the geometry are rather more straightforward this time. The numerical polynomials involved arise directly from the binomial coefficient polynomials, while the space which we have to embed is a projective space.

Proposition 9.1. If $n$ is a natural number and $\alpha(n)$ denotes the sum of its dyadic digits then $w_{2}^{2 n-\alpha(n)}\left(\begin{array}{c}w_{1} w_{2}^{-1} \\ n^{2}\end{array}\right)$ is a homogeneous numerical polynomial.

Proof. The result follows from Proposition 1.9 since the largest exponent of any prime in $n !$ is $\nu_{2}(n !)=n-\alpha(n)$.

Let $\zeta \in \widetilde{K}^{0}\left(C P^{n}\right)$ denote the reduced Hopf bundle, so that $\zeta, \zeta^{2}, \ldots, \zeta^{n}$ is the basis for $\widetilde{K}^{0}\left(C P^{n}\right)$ dual to $\beta_{1}, \beta_{2}, \ldots, \beta_{n}$.

Proposition 9.2. The homomorphism $\left(\zeta^{i}\right)_{*}: K_{0}\left(C P^{n}\right) \rightarrow K_{0}(K)$ maps $\beta_{j}$ to the coefficient of $\left(\begin{array}{l}x \\ i\end{array}\right)$ when $\left(\begin{array}{c}w 1 x \\ j\end{array}\right)$ is expanded as a linear combination of the binomial coefficient polynomials in $x$.

Proof. It is straightforward to verify that $\psi\left(\beta_{j}\right)=\sum_{i=1}^{n}\left(\zeta^{i}\right)_{*}\left(\beta_{j}\right) \otimes \beta_{i}$. The result now follows from the remarks which precede Definition 1.5 on the computation of the coaction on $K_{0}\left(C P^{n}\right)$.

By a result of Mukherjee [37], $C P^{n}$ may be embedded in $S^{4 n-\alpha(n)-1}$ if $n$ is odd, and in $S^{4 n-\alpha(n)}$ if $n$ is even. We choose $d$ so that

$$
2 d \geq \begin{cases}4 n-\alpha(n), & \text { if } \alpha(n) \text { is even, } \\ 4 n-\alpha(n)-1, & \text { if } \alpha(n) \text { and } n \text { are both odd, } \\ 4 n-\alpha(n)+1, & \text { if } \alpha(n) \text { is odd and } n \text { is even. }\end{cases}
$$


This ensures that $C P^{n}$ will embed in $S^{2 d}$. Note that in every case $d \geq 2 n-$ $\alpha(n)$, with equality holding only for $n=1$. Assume now that $n>1$. Let $Y$ denote the complement of $C P^{n}$ in $S^{2 d+1}$. Hence $Y$ is an $S$-dual of $C P^{n}$ and has the homotopy type of a suspension since $C P^{n}$ can be embedded in one dimension less.

Proposition 9.3. If $y_{j} \in \widetilde{K}^{0}(Y)$ is such that $D y_{j}=\beta_{j} \in \widetilde{K}_{0}\left(C P^{n}\right)$ then $\left(y_{j}\right)_{*}$ : $K_{0}(Y) \rightarrow K_{0}(K)$ maps $D\left(\zeta^{i}\right)$ to $w^{d}$ times the coefficient of $\left(\begin{array}{c}x \\ i\end{array}\right)$ in $\left(\begin{array}{c}w^{-1} x \\ j\end{array}\right)$.

Proof. This follows immediately from Propositions 7.6 and 9.2.

Let $M$ denote the hypersurface separating $C P^{n}$ and $Y$. It follows from Proposition 7.3 that the map induced in $K$-theory by the composition

$$
S^{2 d+1} \stackrel{c}{\longrightarrow} S^{1} \wedge M \stackrel{1 \wedge(i \times j)}{\longrightarrow} S^{1} \wedge\left(C P^{n} \times Y\right)
$$

sends the generator of $\widetilde{K}_{0}\left(S^{2 d}\right)$ to $\sum_{i=1}^{n} \beta_{i} \otimes D\left(\zeta^{i}\right)$.

Let $f$ denote the inclusion $C P^{n} \subset C P^{\infty}$ and let $g: Y \rightarrow B U$ represent $y_{n}$. Since $f_{*}\left(\beta_{i}\right)=\left(\begin{array}{c}w \\ i\end{array}\right) \in K_{0}\left(C P^{\infty}\right)$, Proposition 9.3 shows that the map

$$
1 \otimes l: K_{0}\left(C P^{\infty}\right) \otimes K_{0}(B U) \rightarrow K_{0}\left(C P^{\infty}\right) \otimes K_{0}(K) \cong A \otimes A\left[w^{-1}\right]
$$

sends $\sum_{i=1}^{n} f_{*}\left(\beta_{i}\right) \otimes g_{*}\left(D\left(\zeta^{i}\right)\right)$ to $w_{2}^{d}\left(\begin{array}{c}w_{1} w_{2}^{-1} \\ n\end{array}\right)$, where $w_{1}=w \otimes 1$ and $w_{2}=$ $1 \otimes w$. As $Y$ is a suspension, $g_{*}\left(D\left(\zeta^{i}\right)\right)$ is diagonal primitive and the discussion in $\S 5$ shows that the Hurewicz image of the class $[M, f \oplus g]$ is $\frac{1}{n !} \sum_{i=1}^{n} s_{n}^{i} \Gamma_{i} \Sigma_{d-i}$, where $s_{n}^{i}$ is the Stirling number of the first kind [11] defined by the formula $n !\left(\begin{array}{l}w \\ n\end{array}\right)=\sum_{i=1}^{n} s_{n}^{i} w^{i}$, for $n>0$. Thus the class $[M, f \oplus g]$ realizes the element of the form (5.3) corresponding to the polynomial $w_{2}^{d-1}\left(\begin{array}{c}w_{1} w_{2}^{-1} \\ n\end{array}\right)$, which, by Proposition 9.1, is numerical since $d>2 n-\alpha(n)$.

As in $\S 8$ it is easy to see that no new primitive elements are produced by varying $g$.

There is a range of values $2 d$, from $4 n-2 \alpha(n)$ to approximately $4 n-\alpha(n)$, over which $\frac{1}{n !} \sum_{i=1}^{n} s_{n}^{i} \Gamma_{i} \Sigma_{d-i}$ exists in $P_{d} K_{0}(B U)$ but is not known to be stably spherical. Any improved embedding results for $C P^{n}$ would imply that the element was spherical for corresponding values of $d$.

The calculation can be turned on its head to obtain a nonembedding result.

Suppose that there is a subcomplex of $S^{2 d+1}$ of the homotopy type of $C P^{n}$. Let $Y$ be the complement, with $f$ and $g$ as above; then we have seen that the map in $K$-theory induced by the composition

$$
S^{2 d+1} \stackrel{c}{\longrightarrow} S^{1} \wedge M \stackrel{1 \wedge(f \times g)}{\longrightarrow} S^{1} \wedge\left(C P^{\infty} \times B U\right) \rightarrow S^{1} \wedge C P^{\infty} \wedge K
$$

sends the generator of $\widetilde{K}_{0}\left(S^{2 d}\right)$ to $w_{2}^{d}\left(\begin{array}{c}w_{1} w_{n}^{-1} \\ n\end{array}\right)$. But, by the discussion at the end of $\S 2$, this must be a two-variable numerical polynomial. Now the remarks following the proof of Proposition 1.9 show that the bound on $d$ given by 
Proposition 9.1 is the best possible, since $n !\left(\begin{array}{l}w \\ n\end{array}\right)$ is monic. Hence $d \geq 2 n-$ $\alpha(n)$, and $C P^{n}$ does not embed up to homotopy type in $\mathbf{R}^{4 n-2 \alpha(n)-1}$. We can improve this statement by one extra dimension by using an amended version of Proposition 7.6 which applies to embeddings in an even-dimensional sphere.

This result has been obtained by Hubbuck [23]. The fact that $C P^{n}$ does not embed smoothly in $\mathbf{R}^{4 n-2 \alpha(n)}$ was obtained in [7] by methods which ultimately rely on the same calculation with numerical polynomials as ours.

\section{EXAMPLES AND COUNTEREXAMPLES}

In the last two sections we have realized two families of primitive elements in $K_{0}(B U)$. In this section we consider first a result in the opposite direction. We give an example of an element of $P_{*} K_{0}(B U)$ which does not belong to the image of the stable Hurewicz map. We then consider a generalization of the construction of $\S 8$.

Recall that in Example 2.3 we defined elements

$$
g_{r}=g_{r}\left(x_{1}, x_{2}\right) \in P_{2 r+2} K_{0}(B U(2)), \quad r \geq 2 .
$$

We showed there that $g_{r}\left(x_{1}, x_{2}\right)$ was not toroidal. In terms of the $\Gamma_{i}$ we have $g_{r}=\frac{1}{4}\left(\Gamma_{r+2} \Gamma_{r}-\Gamma_{r+1}^{2}\right)$, where here multiplication is induced by Whitney sum. The closest we have come to realizing this element is

$$
\Xi_{r+1, r,(1)}=\frac{1}{2}\left(\Sigma_{r+2} \Sigma_{r}-\Sigma_{r+1}^{2}\right),
$$

which is stably spherical for $r \geq 2$ by Theorem 8.2.

Proposition 10.1. $g_{2}=\frac{1}{4}\left(\Gamma_{4} \Gamma_{2}-\Gamma_{3}^{2}\right)$ does not belong to the image of the Hurewicz map $\pi_{12}^{S}(B U) \rightarrow P_{6} K_{0}(B U)$.

Proof. We localize at the prime 2 and compute with the Atiyah-Hirzebruch spectral sequence $H_{*}\left(B U ; \pi_{*}^{S}\right) \Rightarrow \pi_{*}^{S}(B U)$. In [36] Mosher has studied this spectral sequence for the space $C P^{\infty}$, though his indexing is different; his $E^{r}$ term is our $E^{2 r}$-term for example.

We write $b_{n} \in H_{2 n}\left(C P^{\infty}\right)$ for the generator, so that $H_{*}(B U)$ is the polynomial algebra on $b_{1}, b_{2}, \ldots$. Since the Chern character of $\Gamma_{k}$ is $k ! b_{k}$, we see that $\operatorname{ch}\left(g_{2}\right)=12 b_{4} b_{2}-9 b_{3}^{2}$.

We will show that $12 b_{4} b_{2}-9 b_{3}^{2}$ is not an infinite cycle in the spectral sequence. It will follow that $g_{2}$ is not stably spherical.

Mosher's results and the fact that the differentials are derivations are sufficient to compute up to dimension 12 . We use the standard notation for elements of the stable stem.

By Proposition 5.1 of [36] $d^{2} b_{n+1}=n \eta b_{n}$. Thus $d^{2} b_{5} b_{1}=d^{2} b_{3}^{2}=0$ and $d^{2} b_{4} b_{2}=\eta\left(b_{4} b_{1}+b_{3} b_{2}\right)$, showing that $12 b_{4} b_{2}-9 b_{3}^{2}$ is a cycle in the $E^{2}$-term.

The differential $d^{3}$ is zero since $H_{*}(B U)$ is concentrated in even dimensions. 
By Proposition 5.2 of [36] $d^{4} b_{3}=2 \nu b_{1}, d^{4} 2 b_{4}=2 \nu b_{2}$, and $d^{4} b_{5}=\nu b_{3}$. It follows that $d^{4}\left(12 b_{4} b_{2}-9 b_{3}^{2}\right)=12 \nu b_{2}^{2}-36 \nu b_{3} b_{1}$.

But $d^{2}\left(\eta^{2} b_{3} b_{2}\right)=\eta^{3} b_{3} b_{1}$, so that $36 \nu b_{3} b_{1}$ is zero in $E_{8,3}^{4}$ since $\eta^{3}=4 \nu$.

However, $12 \nu b_{2}^{2}=4 \nu b_{2}^{2}$ is not zero. Certainly $d^{2} \nu b_{2}^{2}=0$, but $4 \nu b_{2}^{2}$ cannot be the image under $d^{2}$ of any element of $E_{10,2}^{2}=H_{10}\left(B U ; \pi_{2}^{S}\right)$, for $\eta^{2} b_{4} b_{1}$ and $\eta^{2} b_{3} b_{2}$ are the only candidates, but $d^{2}$ sends both of them to $4 \nu b_{3} b_{1}$.

In [19] Felali independently obtained the same result and extended the spectral sequence calculations to show that $g_{3}$ is stably spherical. In fact two of us have shown in [44], by more geometric methods, that $g_{r}$ is stably spherical for all $r \equiv 3 \bmod 4$. In view of Example 2.3 this gives examples of elements in the stable homotopy of $B U(2)$ which do not arise from the stable homotopy of the classifying space of a maximal torus.

In the remainder of this section we give some examples of stable homotopy elements which are realized by embedding two-cell complexes. These elements generalize the elements of the form $\Xi_{i, j,(a)}$ which were realized in $\S 8$.

We first make some general remarks about the connection between the $e$ invariant and our construction for elements in the stable homotopy of $B U$ and then apply these remarks to two examples.

Let $\alpha: S^{2 n-1} \rightarrow S^{2 m}$ be a suspension, with $n>m \geq 1$, and let $X_{\alpha}$ denote the mapping cone $S^{2 m} \cup_{\alpha} e^{2 n}$, so that $X_{\alpha}$ is a suspension.

By Corollary 3 of [16], $X_{\alpha}$ embeds up to homotopy type in $S^{2 n+2 j+1}$, with complement $Y_{\alpha}=S^{2 j} \cup_{\beta} e^{2 n+2 j-2 m}$ if $j \geq m$, where $\beta=S^{2 j-2 m} \alpha$. Clearly $Y_{\alpha}$ is also a suspension.

If $e(\alpha)$ is the (complex) $e$-invariant of $\alpha$ we can choose a basis $x_{1}, x_{2}$ for $\widetilde{K}_{0}\left(X_{\alpha}\right)$ such that

$$
\begin{aligned}
& \psi\left(x_{1}\right)=w^{m} \otimes x_{1}, \\
& \psi\left(x_{2}\right)=w^{n} \otimes x_{2}+e(\alpha)\left(w^{n}-w^{m}\right) \otimes x_{1} .
\end{aligned}
$$

If $\xi_{1}, \xi_{2}$ is the dual basis for $\widetilde{K}^{0}\left(X_{\alpha}\right)$ then

$$
\begin{array}{ll}
\left(\xi_{1}\right)_{*}\left(x_{1}\right)=\Sigma_{m}, & \left(\xi_{1}\right)_{*}\left(x_{2}\right)=e(\alpha)\left(\Sigma_{n}-\Sigma_{m}\right), \\
\left(\xi_{2}\right)_{*}\left(x_{1}\right)=0, & \left(\xi_{2}\right)_{*}\left(x_{1}\right)=\Sigma_{n},
\end{array}
$$

in $\widetilde{K}_{0}(B U)$.

Following again the program described in $\S 7$, we let $f: X_{\alpha} \rightarrow B U$ represent $\xi_{1}$ and choose $g: Y_{\alpha} \rightarrow B U$ so that its dual is $x_{2} \in \widetilde{K}_{0}\left(X_{\alpha}\right)$. It follows from Proposition 7.6, and the fact that $Y_{\alpha}$ is a suspension, that

$$
g_{*}\left(D \xi_{1}\right)=e(\alpha)\left(\Sigma_{j}-\Sigma_{n+j-m}\right), \quad g_{*}\left(D \xi_{2}\right)=\Sigma_{j} .
$$

If, as usual, $M$ denotes the hypersurface separating $X_{\alpha}$ and $Y_{\alpha}$, the $K$ theory Hurewicz image of $[M, f \oplus g]$ is

$$
f_{*}\left(x_{1}\right) g_{*}\left(D \xi_{1}\right)+f_{*}\left(x_{2}\right) g_{*}\left(D \xi_{2}\right)=e(\alpha)\left(\Sigma_{n} \Sigma_{j}-\Sigma_{m} \Sigma_{n+j-m}\right) .
$$


If $\alpha$ is the double suspension of the element obtained by applying the $J$ homomorphism to the generator of $\pi_{2 n-2 m-1}(U(m-1))$, whose $e$-invariant is $B_{n-m} /(n-m)$, we obtain the element $\Xi_{m, j,(n-m)}$, if $m \leq 2 m$ and $j \geq m$. We have a special case of Theorem 8.2.

Now if $p$ is an odd prime and $p^{s-1}(p-1)$ divides $k$, then, by Theorem 6.2 of [21], for $i>s$ there is a map

$$
\alpha: S^{2 k+2 i-1} \rightarrow S^{2 i}
$$

which is a suspension and which has $e$-invariant $1 / p^{s}$. Our construction then realizes $\left(1 / p^{s}\right)\left(\Sigma_{k+i} \Sigma_{j}-\Sigma_{i} \Sigma_{k+j}\right)$, if $j \geq i$.

Now $p^{s}$ divides $o(k)$, the denominator of $B_{k} / k$. Thus for sufficiently large $i$ we simply have an integer multiple of the element $\Xi_{i, j,(k)}$. For smaller values of $i$, however, these are, in general, new elements of $\pi_{*}^{S}(B U)$.

The algebra underlying these examples is clear. The polynomial $f(w)=$ $w^{k}-1$ is divisible by $m(k)$ in $A\left[w^{-1}\right]$. If $N$ is a divisor of $m(k)$, the polynomial $(1 / N) w_{1}^{i} w_{2}^{j+k} f\left(w_{1} w_{2}^{-1}\right)$ is numerical if $i$ and $j$ are at least as large as the maximum exponent of any prime occurring in $N$. By choosing $N=p^{s}$, we have a numerical polynomial for smaller values of $i$ and $j$ than if $N=o(k)$. What we have shown is that this algebraic phenomenon is reflected in the geometry.

\section{Conjectures and SPECUlations}

We conclude with a brief discussion of certain conjectures and open questions.

The simplest possibility is provided by

Conjecture 11.1. At odd primes the Hurewicz homomorphism

$$
\pi_{*}^{S}(B U) \rightarrow P_{*} K_{0}(B U)
$$

is an epimorphism.

Of course this is false for the prime 2, as illustrated by Proposition 10.1, but we have no counterexample for any odd prime. In the unlikely event of 11.1 being true, we might consider using $K O$-theory for an improved statement at the prime 2 .

Snaith [49] has shown that there is a stable splitting $B U \simeq \bigvee_{m \geq 1} M U(m)$. Thus it is possible to investigate Conjecture 11.1 in terms of the Hurewicz maps $\pi_{*}^{S}(M U(m)) \rightarrow P_{*} K_{0}(M U(m))$ for each $m$. If $m=1$, we do indeed have an epimorphism, as we saw in $\S 1$.

For general $m$, recall that $P_{*} K_{0}(M U(m))$ is rationally generated by the monomials in the $\Gamma_{k}$ of length $m$, as a subgroup of $P_{*} K_{0}(B U)$. Thus by appealing to the Hattori-Stong theorem, i.e., that

$$
\pi_{2 i+2 m}^{S}(M U(m)) \rightarrow P_{i+m} K_{0}(M U(m))
$$


is an epimorphism in the stable range $i \leq m$, we at least deduce that any element of $P_{r} K_{0}(M U(m))$ consisting of monomials in the $\beta_{k}$ of length $q$ or more is stably spherical so long as $r \leq 2 q$.

The fact that our work has identified two algebraically intricate families of primitives as stably spherical might also be construed as further evidence in favor of Conjecture 11.1.

Similar conjectures can be made after replacing $B U$ by related spaces. For example

Conjecture 11.2. The Hurewicz homomorphism

$$
\pi_{*}^{S}\left(B T^{m}\right) \rightarrow P_{*} K_{0}\left(B T^{m}\right)
$$

is an epimorphism for all $m \geq 1$.

Since this conjecture holds for $m=1$, as noted above, and also for $m=2$ through a considerable range of dimensions [29], it may well be a more likely possibility than 11.1 . Indeed it suggests that we might weaken 11.1 by proposing that $\pi_{*}^{S}(B U(m)) \rightarrow P_{*} K_{0}(B U(m))$ is an epimorphism only at primes larger than $m$.

Some information has also been obtained for the cases $S O / U$ (see [10]) and $U$ (see [20]), while $B S p$ at the prime 2 and $U(n) / T^{m}$ for $m \leq n$ are tempting alternatives. Furthermore, one of us has shown [48] that at odd primes, and after the inversion of certain elements, $\pi_{*}^{S}(B G) /$ Torsion and $P_{*} K_{0}(B G)$ are isomorphic for any compact connected Lie group $G$.

There are several other comments to be made, both algebraic and geometric.

We should stress that the elements realized in $\S 8$ are all quadratic in the $\Sigma_{i}$, and we have no reason to believe that they and the elements of $\S \S 9$ and 10 exhaust the algebraic complexity of $P_{*} K_{0}(B U)$. Worse, if we write $\Xi_{*}$ for the subring of $P_{*} K_{0}(B U)$ generated by the $\Sigma_{k}$ and the elements $\Xi_{i, j, K}$ of Definition 5.7, we have no general information on the extensions of graded rings

$$
\mathrm{Z}\left[\Sigma_{1}, \Sigma_{2}, \ldots\right] \subset \Xi_{*} \subset P_{*} K_{0}(B U),
$$

both of which extensions are finite in each degree. Such information would, perhaps, begin to elucidate the real nature of the involvement of the Bernoulli numbers and the Kummer congruences in the $K$-theory of $B U$.

In fact, open questions also remain about the Kummer congruences themselves. For example, we may ask whether the polynomials $W_{K}(w)$, or rather their analogs when $B_{k} / k$ is replaced by $B_{k} / 2 k$ (see the remarks following Corollary 4.10), are divisible within the ring $A\left[w^{-1}\right]$ of stably numerical polynomials. Certainly they are not for sequences $K$ of length one.

More geometrically, we should point out that we have been forced to work with expressions involving the $\Sigma_{k}$, rather than the potentially simpler $\Gamma_{k}$, by our method of evaluating the effect in $K$-theory of a map $f: Y \rightarrow B U$. Thus, when $Y$ is an iterated Thom complex or its dual, we consider first the induced 
map into $K_{0}(K)$ and then use the assumption that $Y$ is a suspension to read off the required answer as a linear combination of the $\Sigma_{k}$. An extra price to pay for such an assumption is the loss of a dimension. The question thus arises as to which extra primitives, in terms of the $\Gamma_{k}$ as well as the $\Sigma_{k}$, may be realized by this construction when $Y$ is not a suspension; we are only able to evaluate one or two low-dimensional examples.

A more positive feature of our realization technique is that all our stably spherical classes lie in the image of $\pi_{*}\left(\Omega\left(S^{1} \wedge B U\right)\right) \rightarrow \pi_{*}^{S}(B U)$. It is therefore of great interest to understand how much of $\pi_{*}^{S}(B U)$ (modulo torsion) arises in this manner. With a little care, several of our results can be rephrased to yield information on $\pi_{*}\left(\Omega\left(S^{1} \wedge B U\right)\right)$ itself, which may be thought of as the singular hypersurface bordism ring of $B U$. This ring is rich in structure and subtleties and, together with several of the issues raised above, has recently been studied in its own right [40].

For the torsion subgroup of $\pi_{*}^{S}(B U)$, and of the stable homotopy groups of other related spaces, there seems to be no hope for early progress; even for $\pi_{*}^{S}\left(C P^{\infty}\right)$ the complexity is overwhelming [36]. As we remarked in $\S 8$, the results there which relate to the multiplicative interaction between the torsion and the torsion-free part of $\pi_{*}^{S}(B U)$ can certainly be improved.

\section{REFERENCES}

1. J. F. Adams, On the groups $J(X)$. II, Topology 3 (1965), 137-171.

2. On the groups $J(X)$. IV, Topology 5 (1966), 21-71.

3. __ Lectures on generalised cohomology, Lecture Notes in Math., vol. 99, Springer-Verlag, Berlin, 1969, pp. 1-138.

4. __ Stable homotopy and generalised homology, Chicago Lectures in Math., University of Chicago Press, Chicago, London, 1974.

5. J. F. Adams, A. S. Harris, and R. M. Switzer, Hopf algebras of co-operations for real and complex K-theory, Proc. London Math. Soc. 23 (1971), 385-408.

6. J. F. Adams and F. W. Clarke, Stable operations on complex $K$-theory, Illinois J. Math. 21 (1977), 826-829.

7. M. F. Atiyah and F. Hirzebruch, Quelques théorèmes de non-plongement pour les variétés différentiables, Bull. Soc. Math. France 87 (1959), 383-396.

8. __ Vector fields on homogeneous spaces, Proc. Sympos. Pure Math., vol. 3, Differential Geometry, Amer. Math. Soc., Providence, R.I., 1961, pp. 7-38.

9. A. J. Baker, Combinatorial and arithmetic identities based on formal group laws, Lecture Notes in Math., vol. 1298, Springer-Verlag, Berlin, 1987, pp. 17-34.

10. A. J. Baker and N. Ray, Some infinite families of $U$-hypersurfaces, Math. Scand. 50 (1982), 149-166.

11. C. Berge, Principles of combinatorics, Academic Press, New York, London, 1971.

12. J. M. Boardman, Stable and unstable objects for BP-cohomology, preprint, Johns Hopkins, 1986.

13. Z. I. Borevich and I. R. Schafarevich, Number theory, Academic Press, New York, London, 1966.

14. N. Bourbaki, Groupes et algèbres de Lie, Chaps. 7 and 8, Éléments de Mathématiques, fasc. 38, Hermann, Paris, 1975. 
15. F. W. Clarke, Self maps of $B U$, Math. Proc. Cambridge Philos. Soc. 89 (1981), 491-500.

16. G. Cooke, Embedding certain complexes up to homotopy type in Euclidean space, Ann. of Math. 90 (1969), 144-156.

17. M. C. Crabb and K. Knapp, Vector bundles of maximal codegree, Math. Z. 193 (1986), 285296.

18. A. Dold and D. Puppe, Duality, trace and transfer, Proceedings of the International Conference on Geometric Topology, Warsaw, 1978, PWN, Warsaw, 1980, pp. 81-102; also Proc. Steklov Inst. Math. (1984), 85-103.

19. K. S. Felali, Intersection points of immersed manifolds, Thesis, Univ. of Manchester, 1982.

20. V. Franjou, Quelques éléments dans l'homotopie stable du groupe unitaire, Bull. Soc. Math. France 115 (1987), 309-328.

21. B. Gray, On the sphere of origin of infinite families in the homotopy groups of spheres, Topology 8 (1969), 219-232.

22. M. W. Hirsch and B. Mazur, Smoothings of piecewise linear manifolds, Ann. of Math. Studies, no. 80, Princeton Univ. Press, Princeton, N.J., 1974.

23. J. R. Hubbuck, Stable homotopy invariant non embedding theorems in Euclidean space. Bol. Soc. Brasil. Mat. 5 (1974), 195-205.

24. J. P. Hudson, Piecewise linear topology, Benjamin, New York, 1969.

25. J. E. Humphreys, Introduction to Lie algebras and representation theory, Springer-Verlag, New York, Heidelberg, Berlin, 1972.

26. D. Husemoller, Fibre bundles, 2nd ed., Springer-Verlag, New York, Heidelberg, Berlin, 1975.

27. $\mathrm{K}$. Johnson, The action of the stable operations of complex $K$-theory on coefficient groups, Illinois J. Math. 28 (1984), 57-63.

28. N. M. Katz, p-adic L-functions for CM fields, Invent. Math. 49 (1978), 199-297.

29. K. Knapp, Some applications of $K$-theory to framed bordism: e-invariant and transfer, Habilitationsschrift, Bonn, 1979.

30. N. Koblitz, p-adic numbers, p-adic analysis, and zeta-functions, Springer-Verlag, New York, Heidelberg, Berlin, 1977.

31. T. Kubota and H. W. Leopoldt, Eine p-adische Theorie der Zetawerte, J. Reine Angew. Math. 214/215 (1964), 328-339.

32. E. E. Kummer, Über eine allgemeine Eigenschaft der rationalen Entwicklungscoefficienten einer bestimmten Gattung analytischer Functionen, J. Riene Angew. Math. 41 (1851), 368-372; also in Collected Papers, vol. I, Springer-Verlag, Berlin, Heidelberg, New York, 1975.

33. M. Mahowald and N. Ray, A note on the Thom isomorphism, Proc. Amer. Math. Soc. 82 (1981), 307-308.

34. J. P. May, The homology of $E_{\infty}$ ring spaces, Lecture Notes in Math., vol. 533, Springer-Verlag, Berlin, 1976, pp. 69-206.

35. H. Miller, Universal Bernoulli numbers and the $S^{1}$-transfer, Canad. Math. Soc. Conference Proc., vol. 2, Amer. Math. Soc., Providence, R.I., 1982, pp. 437-449.

36. R. E. Mosher, Some stable homotopy of complex projective space, Topology 7 (1968), 179-193.

37. A. Mukherjee, Embedding complex projective spaces, Bull. London Math. Soc. 13 (1981), 323324.

38. J. A. Murdock, Generalised e-invariants and the Chern character, J. London Math. Soc. 71 (1972), 283-300.

39. S. Ochanine and L. Schwartz, Une remarque sur les générateurs du cobordisme complexe, Math. Z. 190 (1985), 543-557.

40. M. D. Peim, The homotopy and $K$-theory of $\Omega \Sigma B U$, Thesis, University of Manchester, 1988.

41. D. C. Ravenel and W. S. Wilson, The Hopf ring for complex cobordism, J. Pure Appl. Algebra 9 (1977), 241-280. 
42. N. Ray, Extensions of umbral calculus I: penumbral coalgebras and generalised Bernoulli numbers, Adv. in Math. 61 (1986), 49-100.

43. N. Ray, Symbolic calculus: a 19 th century approach to $M U$ and BP, Homotopy Theory (E. Rees and J. D. S. Jones, eds.), Proc. Durham Symposium 1985, London Math. Soc. Lecture Note Ser., no. 117, Cambridge University Press, Cambridge, 1987, pp. 195-238.

44. N. Ray and L. Schwartz, Construction d'éléments dans $\pi_{*}^{S}(B U(2))$, Bull. Soc. Math. France 111 (1983), 449-465.

45. E. Rees, Framings on hypersurfaces, J. London Math. Soc. 22 (1980), 161-167.

46. L. Schwartz, Opérations d'Adams en K-homologie et applications, Bull. Soc. Math. France 109 (1981), 237-257.

47. __ K-théorie et homotopie stable, Thesis, Université de Paris-VII, 1983.

$48 \ldots$, K-théorie des corps finis et homotopie stable du classifiant d'un groupe de Lie, J. Pure Appl. Algebra 34 (1984), 291-300.

49. V. P. Snaith, Algebraic cobordism and K-theory, Mem. Amer. Math. Soc. 221 (1979).

50. J. Stallings, The embedding of homotopy types into manifolds, mimeo notes, Princeton University, Princeton, N.J., 1965.

51. K. G. C. von Staudt, De numeris Bernoullianis-commentatio altera, Erlangen, 1845.

52. R. M. Switzer, Algebraic topology-homotopy and homology, Springer-Verlag, New York, Heidelberg, Berlin, 1975.

53. G. W. Whitehead, Elements of homotopy theory, Springer-Verlag, New York, Heidelberg, Berlin, 1978.

Department of Mathematics, The University, Manchester M13 9PL, England

Department of Mathematics and Computer Science, University College Swansea, SwaNSEA SA2 8PP, WALES

Department of Mathematics, The University, Manchester M13 9PL, England

UA 41169 du C.N.R.S., Université de Paris-Sud, Mathématique, bâtiment 425, F-91405 Orsay Cedex, France 